\title{
Differences in Uptake and Intracellular Fate between Bevacizumab and Aflibercept after Repetitive Long-Term Treatment in the Retinal Pigment Epithelium
}

\author{
Laura Borchers Johann Roider Alexa Klettner \\ Department of Ophthalmology, University of Kiel, University Medical Center, Kiel, Germany
}

\section{Keywords}

Bevacizumab · Aflibercept · Retinal pigment epithelium ·

Lamp2 $\cdot$ Age-related macular degeneration

\begin{abstract}
Introduction: Anti-VEGF therapy is repeatedly given for an extended period of time to patients when treated for agerelated macular degeneration. While short-term effects of anti-VEGF agents on retinal pigment epithelial cells have been investigated, the effects of long-term and repeated treatment on these cells are scarce. In this study, we have investigated the effects of anti-VEGF treatment (bevacizum$\mathrm{ab}$ and aflibercept) after long-term, repeated treatment on uptake, storage, and subcellular localization. Methods: Experiments were conducted in primary porcine retinal pigment epithelium (RPE) cells in first passage and in ARPE-19 cell line. Cells were treated with $250 \mu \mathrm{g} / \mathrm{mL}$ bevacizumab, aflibercept, or, as a non-VEGF inhibiting antibody, rituximab once a week for 1 day, 7 days, 4, and 12 weeks. Cell survival was evaluated with methyl thiazolyl tetrazolium assay. Uptake and localization of compounds were investigated with immunofluorescence microscopy. Selective intracellular proteins were stained with specific respective primary antibodies; actin cytoskeleton was stained with phalloidin. For quantitative analysis, intracellular signals were normalized
\end{abstract}

to light intensity and exposure time. Intracellular association with lysosomes (Lamp2) and exosomes (CD63) was also quantified. In addition, subcellular fractions (nucleus, plasma, membrane, and cytoskeleton) were generated and analyzed in Western blot. Results: Weekly treatment up to 12 weeks displayed no toxic effects on RPE cells in any substance tested. Intracellular signal of bevacizumab and aflibercept was strongest after 1 day, decreased after 1 and 4 weeks but increased again after 12 weeks. The signal of intracellular bevacizumab was significantly stronger than of aflibercept. In addition, in primary RPE, aflibercept was significantly more associated with Lamp2, indicating degradation of aflibercept. At all time points, the respective therapeutics could be detected at the cytoskeleton. In primary RPE cells, co-localization with exosome marker CD63 showed a maximum after 1 day for bevacizumab and after 12 weeks for aflibercept. Actin-encapsulated therapeutics can be found at any time point tested. Conclusion: Both bevacizumab and aflibercept display a distinctive time-dependent uptake in the RPE cells and are stored in actin-covered accumulations for extended periods of time. When normalized and quantified, less aflibercept can be found in RPE cells, while more aflibercept is co-localized with Lamp2. Our data suggest that bevacizumab is differently processed by RPE cells than aflibercept.

(c) 2020 S. Karger AG, Basel

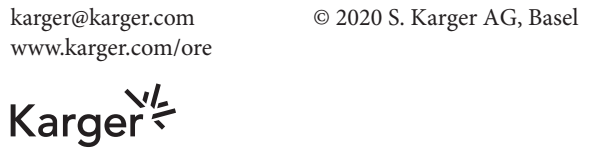

Alexa Klettner

Department of Ophthalmology, University Medical Center

University of Kiel

Rosalind-Franklin-St. 9, DE-24109 Kiel (Germany)

alexakarina.klettner@uksh.de 


\section{Introduction}

Since the approval of ranibizumab (Lucentis ${ }^{\circledR}$, Novartis) for the treatment of age-related macular degeneration (AMD) in 2007, anti-VEGF therapy has become the standard treatment option of exudative AMD [1]. As the treatment is not curative, injections are given repeatedly over an extended period of time, sometimes for several years [2]. The euphoria of the first years has by now been replaced by routine and the realization that the initial gain in visual acuity is usually lost in long-term treatment [2]. A general observation that has been made is appearances of geographic atrophy in long-term treated retina. It has been suggested that the exposure of the retinal pigment epithelium (RPE) to the VEGF antagonists may be responsible for geographic atrophy $[3,4]$. However, this is under debate [5].

We and others have previously shown that the VEGF-inhibitors interact with RPE cells [6-11]. While we and others found no direct toxicity, VEGF-inhibitors can interfere with RPE function and are taken up and stored by RPE cells, provided that they contain an Fc-fragment $[7,12,13]$. The uptake results in transport via Myosin $7 \mathrm{a}$ and intracellular accumulation of the anti-VEGF compounds in some, but not all cells, usually associated with or encapsulated by actin filaments [8, 9]. However, these experiments have been done after exposure to bevacizumab or aflibercept for a maximum of 7 days, not reflecting repeated administration over an extended period of time.

Repetitive long-term treatment is hardly covered in research concerning possible effect of anti-VEGF treatment and could differ profoundly to what is found in short-term experimentation. Indeed, a major drawback of in vitro research is that it usually is limited to short incubation periods, ranging from hours to 1 day, while in vivo, the interaction between the tissue and the compounds is a long-term interaction. We have recently established a model of repetitive long-term treatment with VEGF antagonists for up to 12 weeks, which also takes into account that the anti-VEGF inhibitor is diminished and re-administered during the time course [14]. In this model, we can test whether the observed uptake and storage of anti-VEGF compounds is merely a short-term event or relevant for long-term interaction and whether it shows effects on anti-VEGF availability. In this study, we have investigated the uptake and intracellular localization of bevacizumab and aflibercept, and the nonVEGF binding antibody rituximab, after treatment for up to 12 weeks.

\section{Materials and Methods}

\section{Primary RPE Cell Culture}

Primary RPE cells were prepared from porcine eyes as previously described $[15,16]$. In brief, the eyes of pigs were obtained no later than $4 \mathrm{~h}$ after death; they were cleaned and the anterior parts of the eye, as well as the vitreous and retina, were removed. RPE cells were harvested by trypsin digestion. Cells were cultivated in Dulbecco's Modified Eagle's Medium (DMEM, PAA) supplemented with penicillin/streptomycin (1\%), L-glutamine, amphotericin $\mathrm{B}(0.5 \mathrm{mg} / \mathrm{mL})$, HEPES $(25 \mathrm{~mm})$, sodium-pyruvate $(110$ $\mathrm{mg} / \mathrm{mL}$ ) (all PAA), and 10\% fetal calf serum (LINARISblue, Wertheim-Bettingen, Germany). Confluent RPE cells of first passage (directly harvested) were used. All experiments were carried out in 12 -well plates. For microscopy, cells were cultivated until confluence on collagen-coated (Collagen A, Biochrom, Berlin, Germany) coverslips $(21 \times 26 \mathrm{~mm}$, Menzel GmbH, Braunschweig, Germany), as previously described [9].

\section{ARPE-19 Cells}

ARPE-19 cells (ATCC, Wesel, Germany), an immortal human RPE cell line [17], were cultivated in Hyclone DMEM (GE Healthcare, München, Germany), supplemented with penicillin/streptomycin (1\%), nonessential amino acids (1\%) (all Biochrom, Berlin, Germany), and $10 \%$ fetal calf serum (LINARISblue). For all experiments, confluent cells were used. All experiments were carried out in 12-well plates except for subcellular fractioning, for which ARPE-19 cells were cultured in flasks (T75, Sarstedt, Nümbrecht, Germany). For microscopy, cells were cultivated on collagen-coated (Collagen A, Biochrom) coverslips $(21 \times 26 \mathrm{~mm}$, Menzel $\mathrm{GmbH})$, as previously described [9].

\section{Treatment of Cells}

Confluent primary RPE cells or ARPE-19 cells were treated with $250 \mu \mathrm{g} / \mathrm{mL}$ bevacizumab (Avastin ${ }^{\circledR}$, Roche, Mannheim, Germany), aflibercept $\left(\right.$ Eylea $^{\circledR}$, Bayer, Leverkusen, Germany), or, as a nonVEGF inhibiting biological, rituximab (MabThera ${ }^{\circledR}$, Roche), for 1 day, 7 days, 4 weeks, and 12 weeks. For cells incubated for 7 days or longer, cells were treated with the respective compound for 4 days. After this time, medium was exchanged without any application of the respective compound, and the cells were left untreated for 3 days. For cells incubated for 4 or 12 weeks, medium was exchanged after these 3 days and the inhibitor was again supplied. This treatment was conducted once a week, every week of the treatment.

\section{MTT Assay}

Methyl thiazolyl tetrazolium (MTT) assay [18] was performed as described previously [19]. In brief, cells were stimulated with the respective substance for the indicated time periods as described above, medium was discarded, and cells were washed with PBS, incubated for $2 \mathrm{~h}$ with MTT (Sigma-Aldrich/Merck, Darmstadt, Germany), and dissolved in DMEM without phenol red (GE Healthcare) $(0.5 \mathrm{mg} / \mathrm{mL})$. After removal of MTT, dimethyl sulfoxide (Roth, Karlsruhe, Germany) was added, the plates were shaken for $5 \mathrm{~min}$, and adsorption was measured at $555 \mathrm{~nm}$ with Elx800 (BioTek, Bad Friedrichshall, Germany). Control was set as $100 \%$.

\section{Subcellular Fractioning}

Subcellular fractioning was conducted as previously described [8]. In brief, the ProteoExtract ${ }^{\circledR}$ Subcellular Proteome Extraction 
Kit was used (Calbiochem, Darmstadt, Germany) according to the recommended protocol with modifications. ARPE-19 cells were treated as described above for 4 and 12 weeks. Cells were harvested in ice-cold PBS (Biochrom), centrifuged at $1,300 \mathrm{~g}$ at $4^{\circ} \mathrm{C}$ for $7 \mathrm{~min}$, and the pellet was resuspended in wash buffer. After washing and centrifugation at $325 \mathrm{~g}$ and $4^{\circ} \mathrm{C}$ for $15 \mathrm{~min}$, washing was repeated. All extraction buffers were supplemented with proteinase inhibitors. Cold extraction buffer I was used for extraction of cytosolic proteins. After centrifugation at $1,025 \mathrm{~g}$ and $4^{\circ} \mathrm{C}$ for $15 \mathrm{~min}$, the supernatant was harvested. To extract membrane/organelle proteins from the pellet, extraction buffer II was used. After incubating the cells for $30 \mathrm{~min}$ at $4^{\circ} \mathrm{C}$ and centrifuging at $6,000 \mathrm{~g}$ and $4^{\circ} \mathrm{C}$ for $15 \mathrm{~min}$, the supernatant was harvested. To further extract nuclear proteins from the remaining pellet, ice-cold extraction buffer III (supplemented with benzonase) was added, incubated for 10 $\min$ at $4^{\circ} \mathrm{C}$, and centrifuged at $6,800 \mathrm{~g}$ and $4^{\circ} \mathrm{C}$ for $10 \mathrm{~min}$. The proteins were harvested in the supernatant. Finally, the cytoskeletal proteins were extracted from the remaining pellet with extraction buffer IV. All supernatants were kept on ice and were centrifuged at $17,000 \mathrm{~g}$ and $4^{\circ} \mathrm{C}$ for $10 \mathrm{~min}$, except for the supernatant of the cytoskeletal proteins. The resulting supernatants were transferred to new tubes and stored at $-80^{\circ} \mathrm{C}$ until further evaluation.

\section{Western Blot}

Western blot was conducted as previously described with modifications [8]. In brief, proteins were separated with reducing SDSPAGE, using a $12 \%$ acrylamide (BioRad, Munich, Germany) gel. After blotting, the PVDF-membrane (Roth) was blocked with $4 \%$ skimmed milk (Roth) in Tris-buffered saline with $0.1 \%$ Tween (Merck) for at least $1 \mathrm{~h}$ at room temperature. The blot was treated with the appropriate first antibody (Anti-IgG, Sigma-Aldrich, I-1011; Anti-Hsp70, Santa Cruz, sc-66048; Anti-Calnexin, Santa Cruz, sc-11397; Anti-c-Jun, Cell Signaling Technologies, Denver, CO, USA, \#9165; Anti-Vimentin, Santa Cruz, sc-5565) in 4\% skimmed milk in Tris-buffered saline with $0.1 \%$ Tween overnight at $4^{\circ} \mathrm{C}$. After washing, the blot was incubated with an appropriate HRP-linked antibody (Anti-goat, Biomol, Hamburg, Germany, \#33922; Anti-rabbit, Cell Signaling Technologies, \#7074; Antimouse, Cell Signaling Technologies, \#7076) in 4\% skimmed milk in Tris (Sigma-Aldrich) buffered saline with $0.1 \%$ Tween (Merck). Following the final washing, it was incubated with Immobilon chemiluminescence reagent (AmershamTM, GE Healthcare, Buckinghamshire, UK), and the signal was detected with MF-ChemiBis 1.6 (Biostep, Jahnsdorf, Germany).

\section{Immunofluorescence Studies}

Primary porcine RPE cells or ARPE-19 cells were treated as described above. Immunofluorescence studies were conducted as described elsewhere with modification [9]. In brief, cells were washed with PBS and fixed in 3\% PFA (Merck). Permeabilization was done in $0.1 \%$ Triton $\mathrm{X}$ (Roth) for $5 \mathrm{~min}$. After Triton $\mathrm{X}$ was removed, coverslips were washed twice with PBS and then blocked using Roti $^{\circledR}$-Immunoblock Solution (Roth) at room temperature. Primary antibody (Lamp2, Santa Cruz, sc-8100; CD63, Santa Cruz, $\mathrm{H}-193)$ was diluted in Roti-Immunoblock Solution, and added and incubated overnight at $4^{\circ} \mathrm{C}$ in a humid chamber. Cells were washed 3 times with PBS, and the corresponding secondary antibody (goat-anti-human AlexaFluor 555, ThermoFisher A-21433; donkey-anti-goat, AlexaFluor 647, ThermoFisher A-21447), diluted in Roti-Immunoblock $(1: 1,000)$ with $0.4 \mu \mathrm{M}$ bisbenzimide H (Sigma-
Aldrich) was added for $1 \mathrm{~h}$ at room temperature. To visualize actin filaments, Atto488-phalloidin (Sigma-Aldrich) was added. Cover slides were mounted after washing with PBS and aqua dest. Coverslips were examined using Zeiss Imager M2 microscope together with the Zeiss ApoTome (Carl Zeiss Microscopy GmbH, Germany). The microscope was fitted with the Zeiss AxioCam MRm Camera (Carl Zeiss). All images were analyzed with ZenPro2 Software (Carl Zeiss).

\section{Quantitative Evaluation of Immunofluorescence}

To objectify the results of the immunofluorescence microscopy, ZenPro2 software was used. In order to evaluate the intracellular amount of bevacizumab, aflibercept, and rituximab in an objective way, the total intensity over 1 picture of the channel of the antagonists was measured. To delete unspecific signals, the mean intensity of the same channel measured in the control samples was subtracted from the intensity of the treated samples. To be able to compare pictures with a different exposure time, the corrected intensity was divided by the exposure time. To describe the co-localization between the antagonists and respective vesicle marker (Lamp2, CD63), we counted all dots representing the specific marker and those which were co-localized with an antagonist. The co-localizations were then given in percentage of the total amount of the marker.

\section{Statistics}

All experiments have been repeated at least 3 times in independent experiments. Graphs depict mean and standard deviation. Statistical analysis was conducted with Student's $t$ test using Excel. A value of $p<0.05$ was considered significant.

\section{Results}

\section{Cell Viability}

Cell viability, after repeated treatment with $250 \mu \mathrm{g} / \mathrm{mL}$ aflibercept, bevacizumab, or rituximab, was tested in primary RPE and ARPE-19 cells for 1 day, 7 days, 4, and 12 weeks of treatment. Neither cell type showed any significant cell death at any time point tested (Fig. 1a, b).

\section{Intracellular Intensity}

Intracellular bevacizumab, aflibercept, and rituximab were assessed after 1 day, 7 days, 4, and 12 weeks in immunofluorescence. In order to quantify the data, light intensity was normalized to control and exposition time (corrected light intensity). In primary RPE (Fig. 2), corrected light intensity for bevacizumab and for aflibercept was highest after the first day of application and significantly reduced at later time points. A direct comparison of the signals for bevacizumab and aflibercept revealed that the intensity for bevacizumab was significantly higher at every time point assessed. As an IgG control which has no binding affinity for VEGF, rituximab was also tested. In rituximab treated cells, the signal significantly de- 
creased after 7 days and 4 weeks while the difference between the signals at 1 day and 12 weeks was not significant. When directly compared with bevacizumab, the signal for rituximab was significantly less for all time points except 12 weeks. The differences between aflibercept and rituximab were not significant.

For ARPE-19 cells (Fig. 3), corrected light intensity for bevacizumab was highest at the first day of application and significantly reduced at 7 days and 4 weeks, but not at 12 weeks. Corrected light intensity for aflibercept and rituximab was highest at the first day of application and significantly reduced at all later time points. However, all 3 compounds showed an increase of the signal after 12 weeks. A direct comparison of the signals for bevacizum$\mathrm{ab}$ and aflibercept revealed that the intensity for bevacizumab was significantly higher at 7 days and 4 weeks. When rituximab was directly compared with bevacizum$\mathrm{ab}$, the signal was significantly higher at 1 day, but lower at 7 days. Taken together, these data generally show a strong signal at the first day, a strong reduction of the signal after 7 days and 4 weeks, and an elevation of the signal after 12 weeks, indicating in general mechanism in the cells. Also, the signal for bevacizumab was generally significantly higher than the signal for aflibercept, indicating a reduced uptake or elevated degradation of aflibercept, similar to what is seen in primary cells. Of note, when

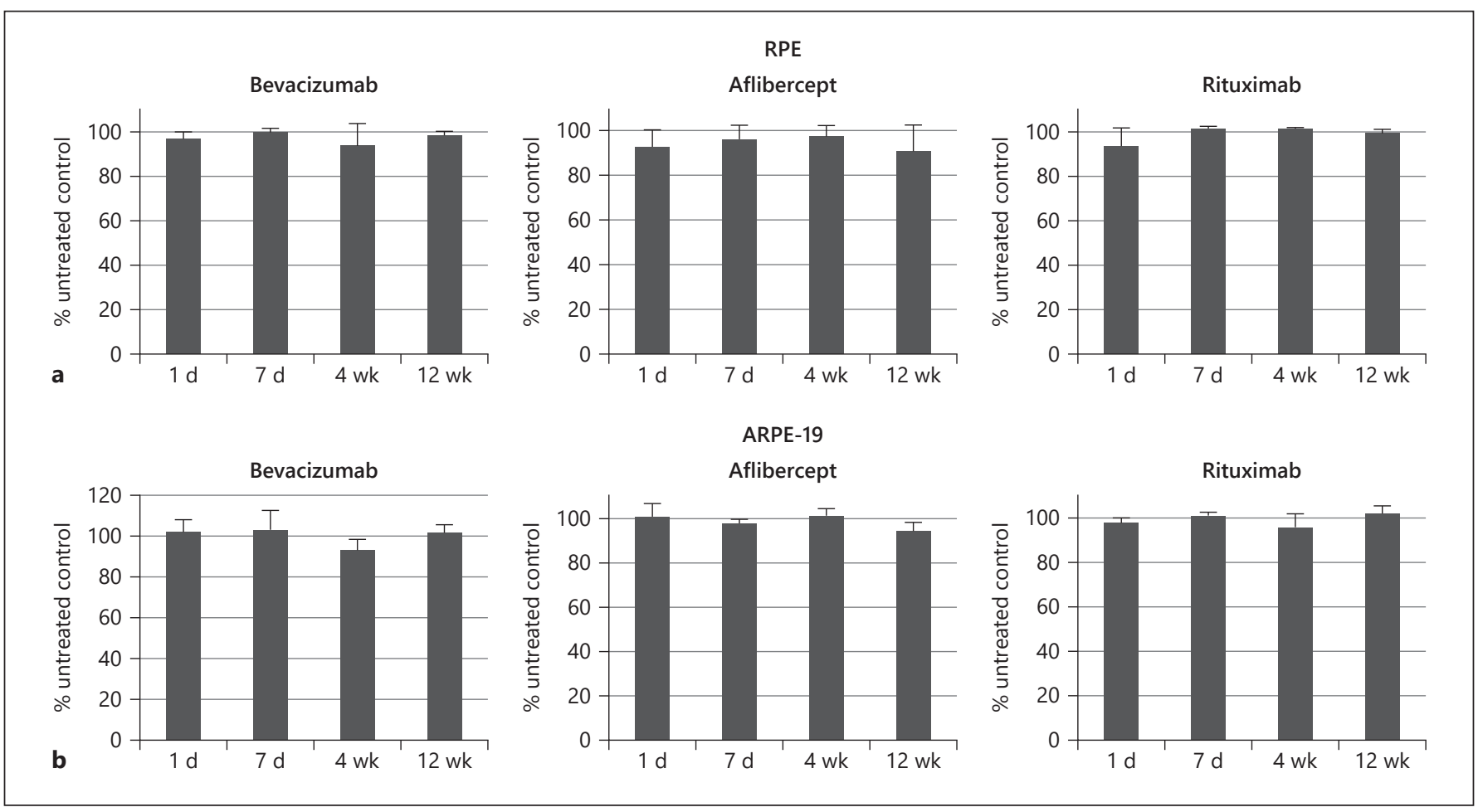

Fig. 1. Influence of tested compounds on cell viability. Influence on cell survival was investigated after treatment with $250 \mu \mathrm{g} / \mathrm{mL}$ bevacizumab, aflibercept, or rituximab for 1 day, 7 days, 4, and 12 weeks in primary RPE cells (a) and ARPE-19 cells (b). Results were normalized to untreated control. No influence on cell viability could be detected for any substance in either of the cell types tested. Significance evaluated with Student's $t$ test. d, day; wk, week; RPE, retinal pigment epithelium.

Fig. 2. Intracellular compound, quantified from immunofluorescence in primary RPE. Comparison of signal intensity of respective compounds at different time points (a), comparison of signal intensity between compounds (b). Generally, the signal reduces over 7 days and 4 weeks to increase again after 12 weeks. Also, bevacizumab generally shows a higher intensity than aflibercept or rituximab. c Exemplary immunofluorescence pictures. Light signal was normalized to control and exposure time. Blue $=$ cell nucleus, red $=$ respective compound. ${ }^{+} p<0.05$; ${ }^{++} p<0.01 ;{ }^{+++} p<0.001$. Significance evaluated with Student's $t$ test. Afli, aflibercept; Beva, bevacizumab; Ritu, rituximab; d, day; wk, week; RPE, retinal pigment epithelium.

(For figure see next page.) 


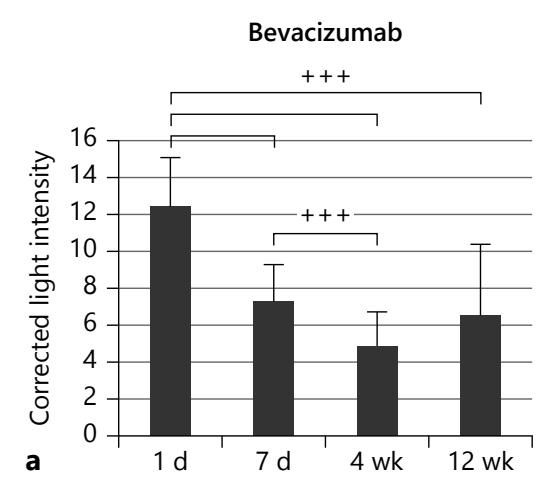

Comparison of time points (primary RPE)
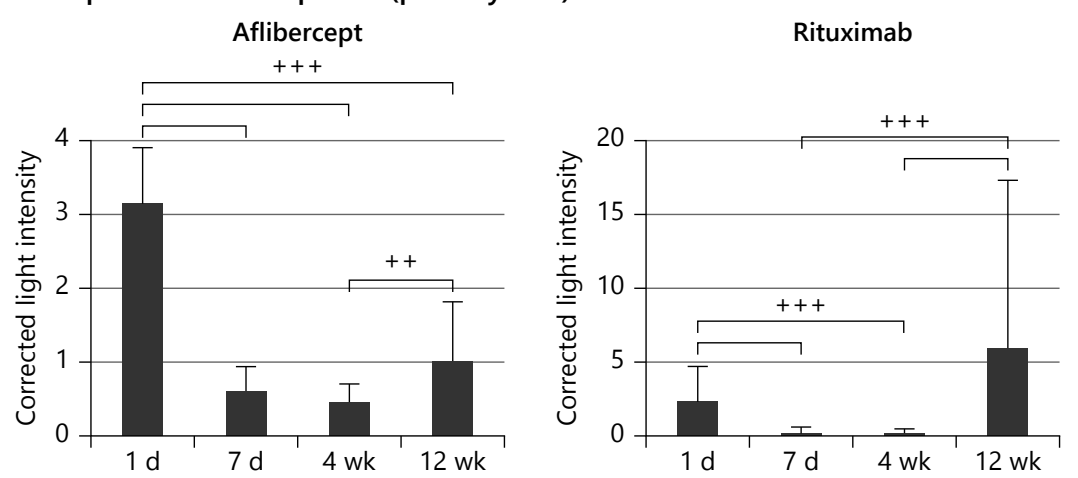

Comparison of compounds (primary RPE)

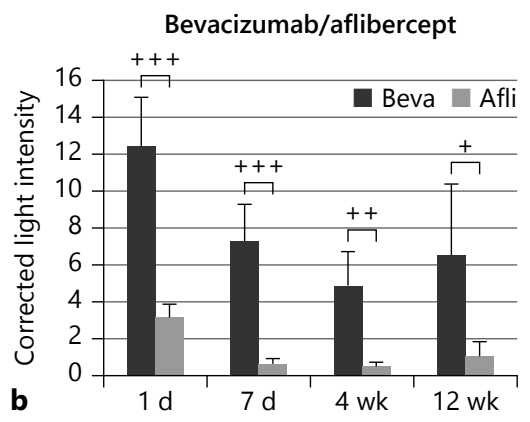
Bevacizumab/rituximab

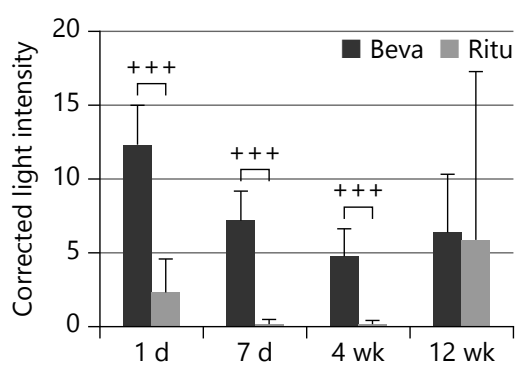

Example pictures primary RPE
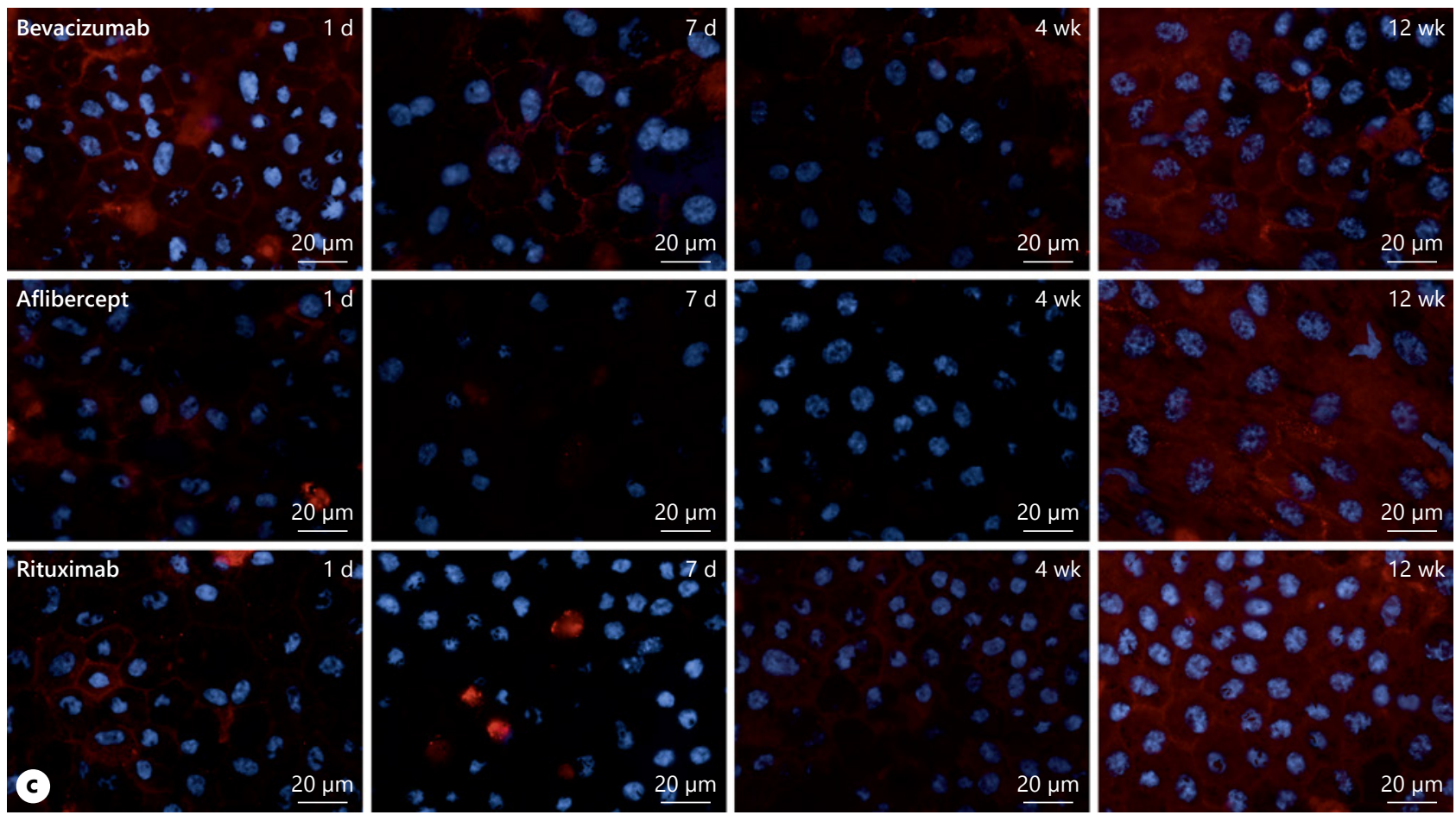

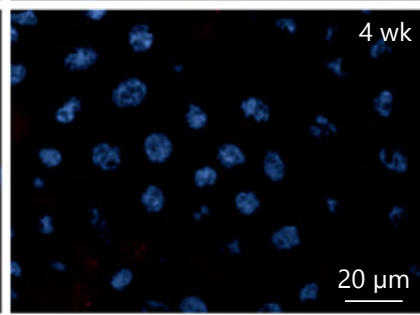

Aflibercept/rituximab
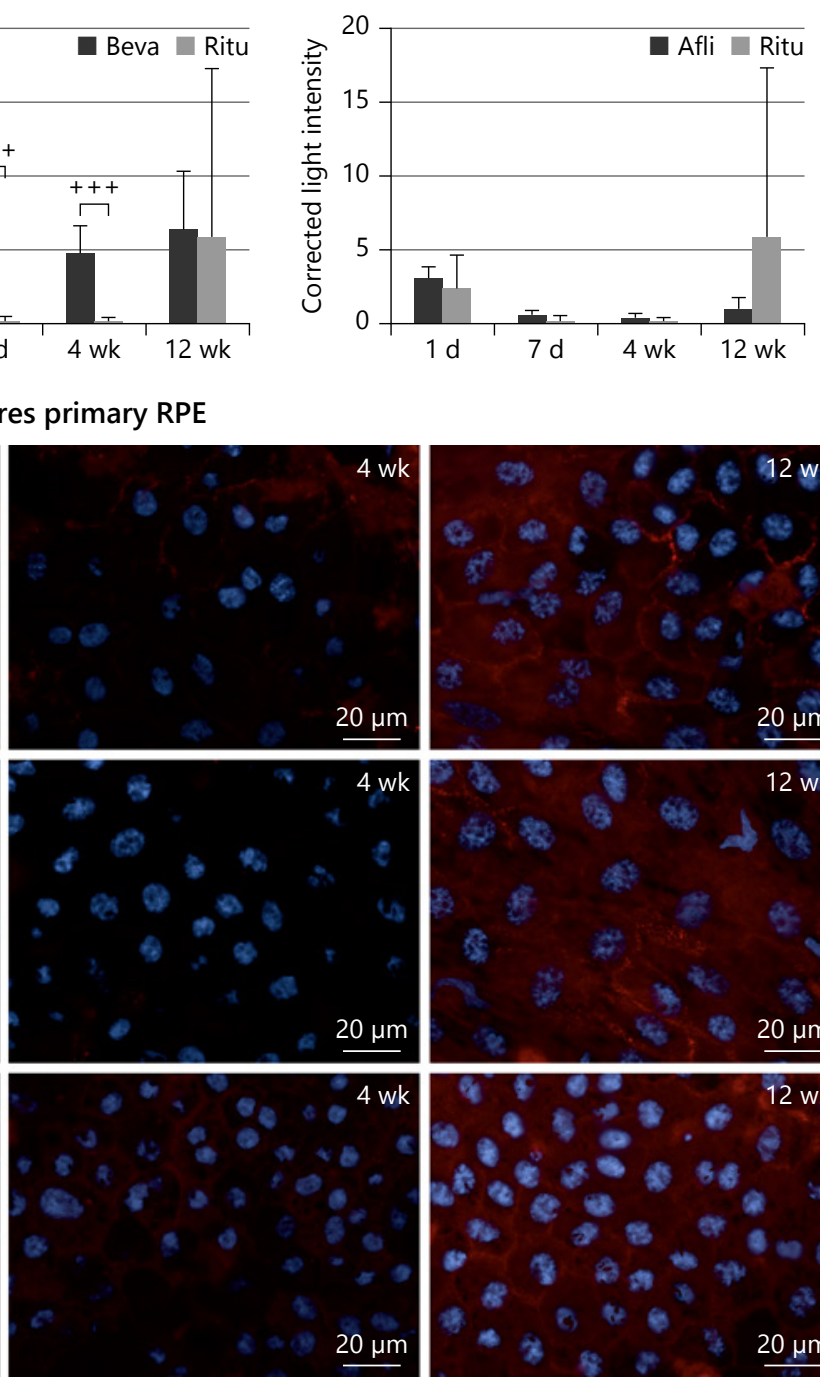

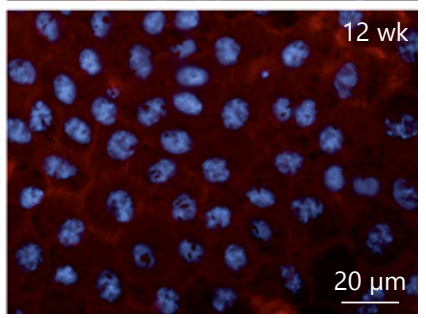


Comparison of time points (ARPE-19)

Bevacizumab

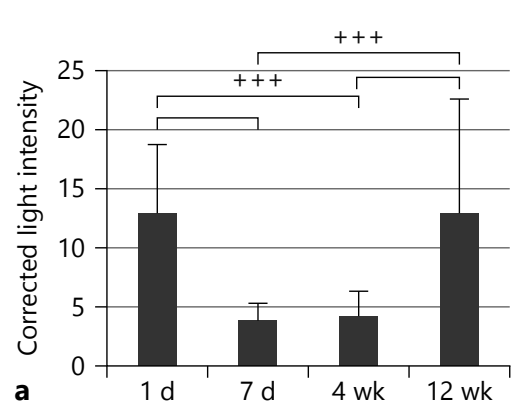

Bevacizumab/aflibercept

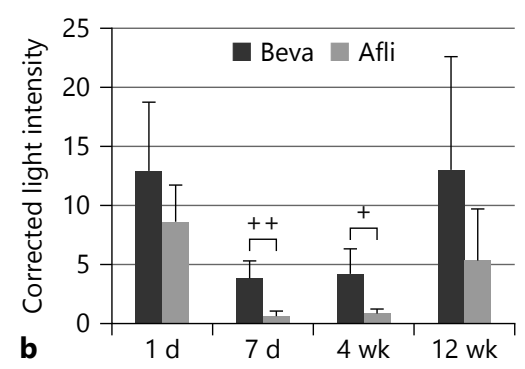

Aflibercept

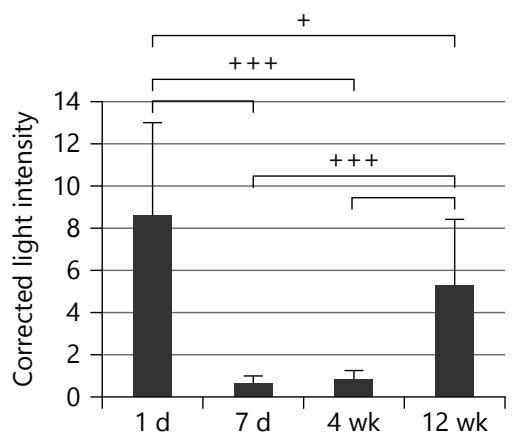

Comparison of compounds (ARPE-19) Bevacizumab/rituximab

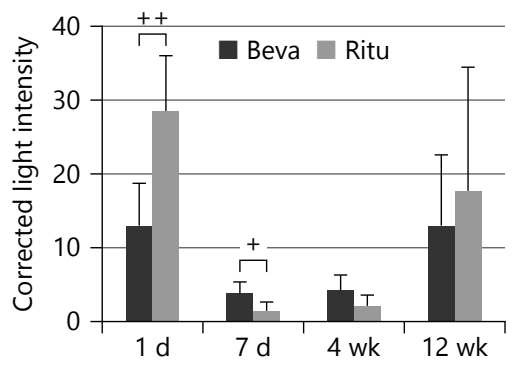

Example pictures (ARPE-19)
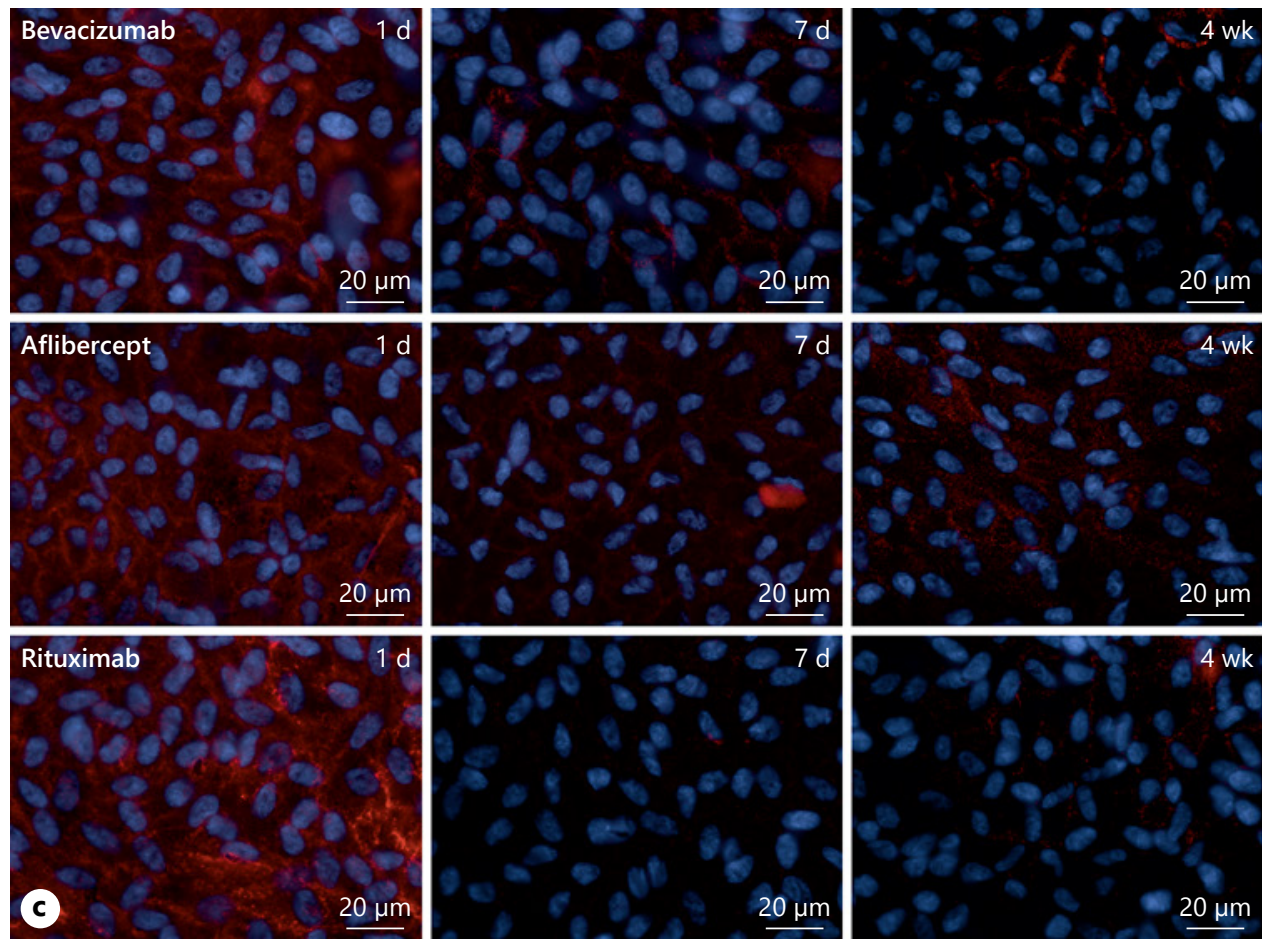
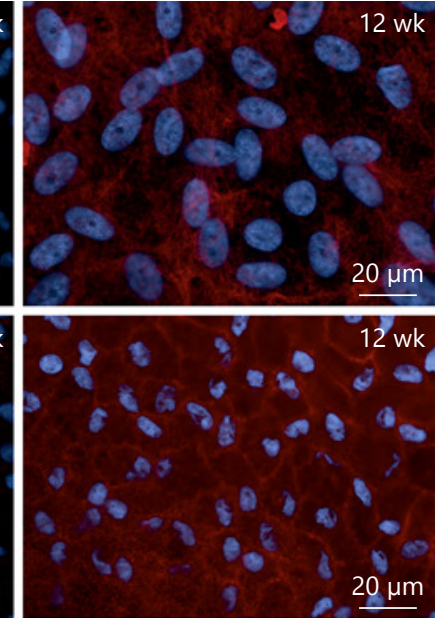

Aflibercept/rituximab
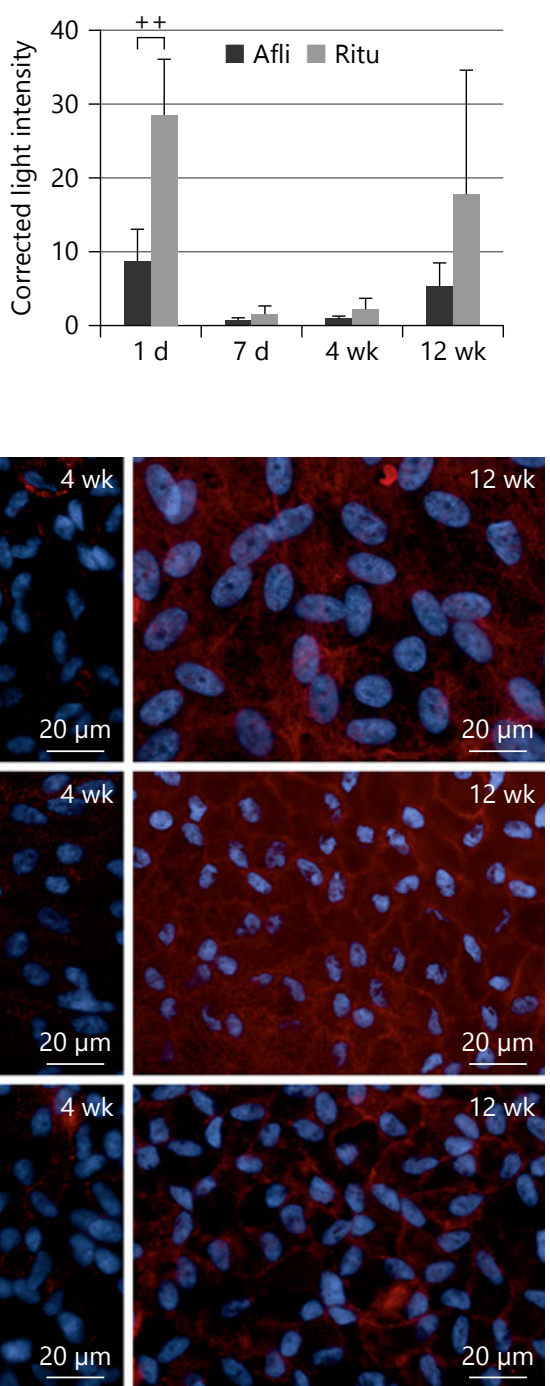

3

(For legend see next page.) 
comparing the cell types, ARPE-19 cells generally showed more signal for aflibercept and rituximab (at 1 day and 12 weeks) compared to primary RPE cells, while the signals for bevacizumab were almost identical.

\section{Intracellular Localization}

\section{Cytoskeleton}

In order to investigate the intracellular localization of bevacizumab, aflibercept, and rituximab at the different time points, a subcellular fractionation was done.
As this method demands high amounts of cells which cannot be provided by primary RPE cells, only ARPE19 cells were used for these experiments. We have previously shown that all 3 substances are mainly found in the cytoskeleton fraction after 1 day of incubation [9]. In this study, we concentrated on 4 and 12 weeks of incubation. After 4 weeks, bevacizumab was mainly found in the cytoskeletal fraction but displayed a more diffuse distribution after 12 weeks. Aflibercept was found in the fraction of the cytoskeleton and of the cell nucleus

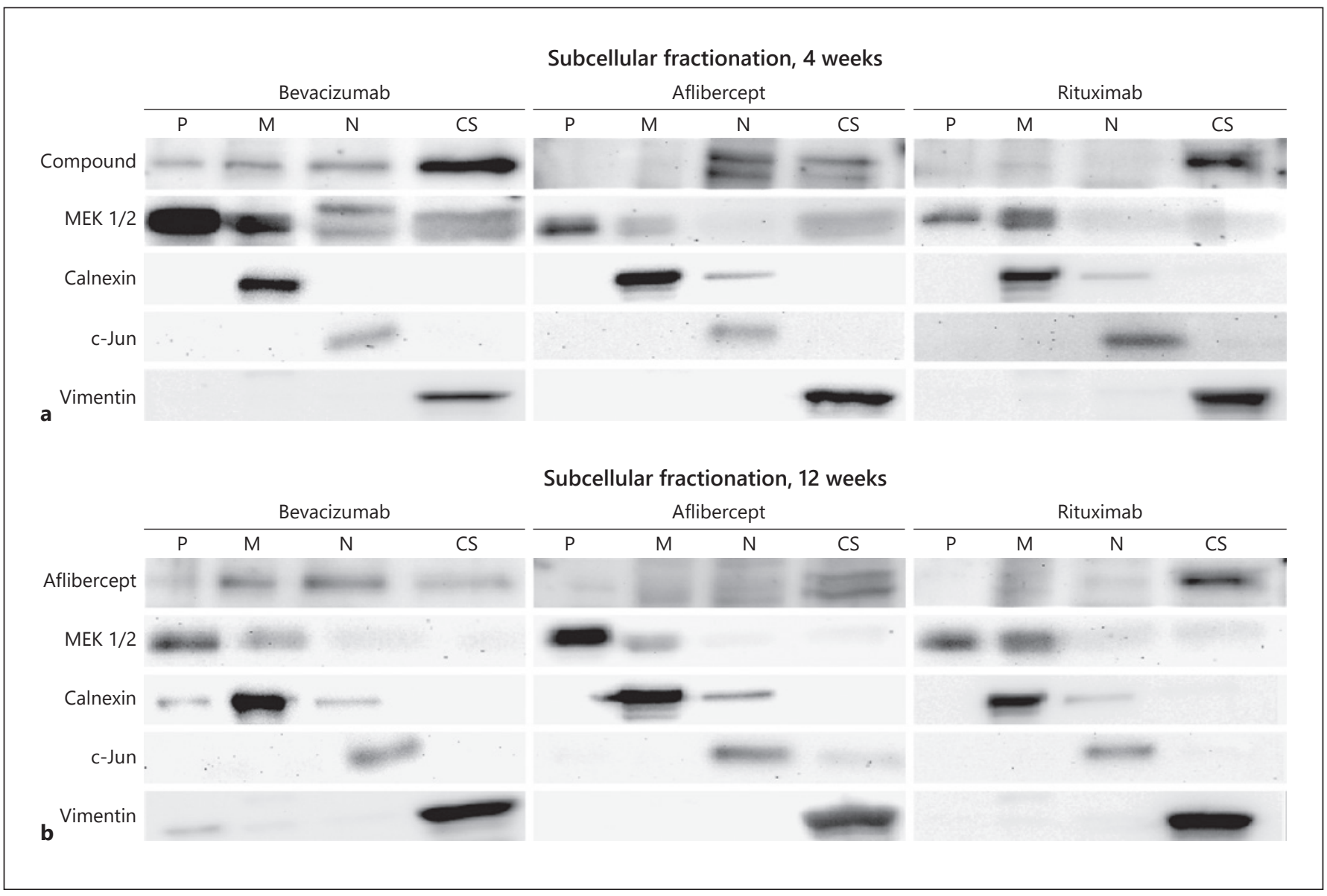

Fig. 4. Subcellular fractionation in ARPE-19 cells. After 4 weeks (a), after 12 weeks (b). Cells were divided into P, M, N, and CS. Exemplary blots for each compound with indicator blots for each fraction (MEK1/2: plasma; Calnexin: membrane; c-Jun: nucleus; vimentin:

Fig. 3. Intracellular compound, quantified from immunofluorescence in ARPE-19 cells. Comparison of signal intensity of respective compounds at different time points (a), comparison of signal intensity between compounds (b). Generally, the signal reduces over 7 days and 4 weeks to increase again after 12 weeks. Also, bevacizumab generally shows a higher intensity than aflibercept. cytoskeleton) are shown. Aflibercept and rituximab show a more pronounced localization to the cytoskeletal fraction than bevacizumab after long-term incubation. P, plasma; M, membrane; N, nucleus; CS, cytoskeleton; RPE, retinal pigment epithelium.

c Exemplary immunofluorescence pictures. Light signal was normalized to control and exposure time. Blue = cell nucleus, red = respective compound. ${ }^{+} p<0.05 ;{ }^{++} p<0.01 ;{ }^{+++} p<0.001$. Significance evaluated with Student's $t$ test. Afli, aflibercept; Beva, bevacizmab; Ritu, rituximab; d; day; wk, week; RPE, retinal pigment epithelium. 


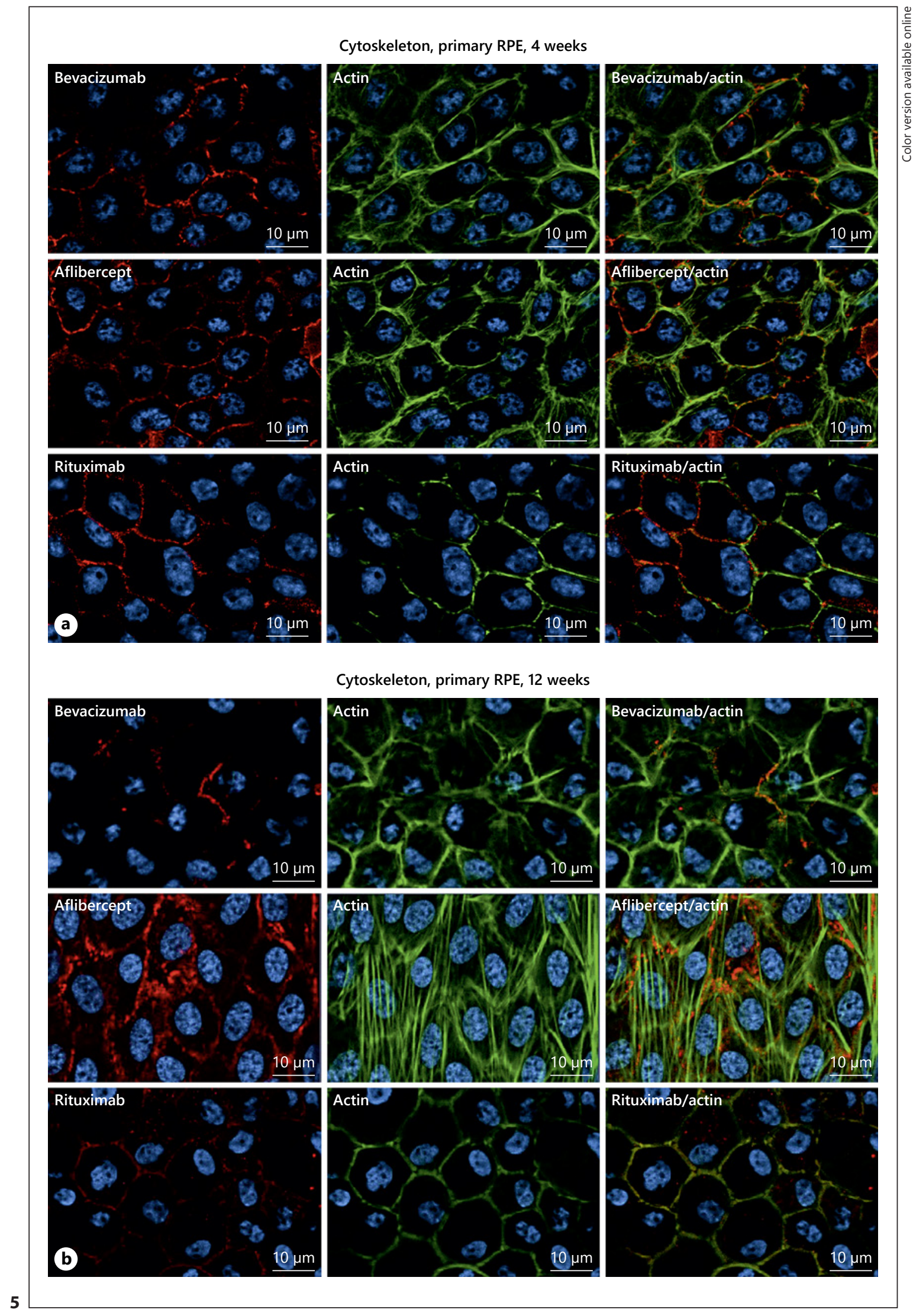

(For legend see next page.) 
after 4 weeks and mainly in the cytoskeletal fraction after 12 weeks. Rituximab was mainly found in the cytoskeletal fraction (Fig. 4). Taken together, while the pattern of distribution displayed variation after 4 and 12 weeks, depending on the compound, all therapeutics were found in the cytoskeletal fraction at any time tested. This correlates with our findings in the immunofluorescence, which shows an alignment of the therapeutics with the actin cytoskeleton at any time point for both primary RPE (Fig. 5) and ARPE-19 cells (Fig. 6).

Of interest, for all compounds and at all time points, actin-coated encapsulations could be found, which was more pronounced in primary RPE cells. When encapsulation of bevacizumab was quantified for size, overall area, or number in primary RPE cells, generally an increase until 4 weeks and a significant decrease between 4 and 12 weeks could be seen. For aflibercept, there was a significant increase in medium size at day 7 , while overall area and number were significantly reduced after 4 and 12 weeks. For rituximab, no statistically significant changes of the medium size were found, while number and overall area were significantly reduced after 12 weeks (Fig. 7).

In ARPE-19 cells (Fig. 8), a significant increase in medium size of the encapsulations of bevacizumab could be found at day 7 and a significant reduction of the number after 12 weeks. Regarding aflibercept, the medium size of the encapsulation was reduced at 4 and 12 weeks (Fig. 8). Overall area or number did not show any differences during the time course. Concerning rituximab, the medium size and the overall area of the encapsulations increased significantly at week 4 . Taken together, encapsulations were found for all compounds at all time points.

\section{Lysosomes/Lamp2}

We have investigated the co-localization of Lamp2, a marker of lysosomes, with bevacizumab, aflibercept, and rituximab. We have quantified the number of distinct colocalization signals between Lamp2 and the respective compounds and normalized it to the number of Lamp2 signals per cell. For primary RPE (Fig. 9), the relative number of co-localizations of bevacizumab was signifi-

Fig. 5. Compounds and cytoskeleton in primary RPE cells. Depicted are exemplary pictures of bevacizumab, aflibercept, and rituximab (red) and the actin cytoskeleton (green) after 4 weeks (a) and 12 weeks (b) of treatment. Co-localizations are seen as a yellow/orange signal. At all time points, the compounds show alignments with the cytoskeleton. Cell nuclei are depicted in blue. RPE, retinal pigment epithelium.

Anti-VEGF Long-Term Uptake cantly lower compared to aflibercept ( 7 days and 4 weeks) and rituximab (all time points). On the other hand, the relative number of co-localizations was almost identical in aflibercept and rituximab treated cells. This correlates well with our data regarding signal intensity, which shows that the signal of bevacizumab was significantly stronger than aflibercept and rituximab, while aflibercept and rituximab did not differ in their signal.

For ARPE-19 cells (Fig. 10), no significant differences between the relative numbers of co-localization of Lamp2 with bevacizumab and aflibercept were detected. For rituximab, we found a higher relative number of co-localization for bevacizumab compared to rituximab (1 day and 4 weeks). When comparing aflibercept with rituximab, no significant difference was found, except at day 1. Again, these data correlate well with our findings of signal intensity, as the signal for aflibercept and rituximab, but not for bevacizumab was higher in ARPE-19 cells compared to primary RPE cells. Therefore, our data suggest that the relative signal we obtain may be related to lysosomal degradation in the respective cell.

\section{Exosomes/CD63}

We have investigated the co-localization of CD63, a marker of exosomes, with bevacizumab, aflibercept, and rituximab. We have quantified the number of distinct colocalization signals between CD63 and the respective compounds and normalized it to the number of CD63 signals per cell. In primary RPE cells, bevacizumab was found co-localized with CD63 mainly after 1 day, while aflibercept displayed the strongest co-localization after 12 weeks. For rituximab, most co-localizations were found after 7 days.

In primary RPE cells (Fig. 11), significantly more bevacizumab was co-localized with CD63 compared to aflibercept after 1 day. However, after 4 and 12 weeks of treatment, there was significantly more aflibercept co-localized with CD63 than bevacizumab. Considering rituximab, bevacizumab was significantly more co-localized after 1 day and 12 weeks of treatment, while at 7 days, there was significantly more rituximab than bevacizumab co-localized with CD63. Comparing aflibercept with rituximab, we find more rituximab co-localized with CD63 after 7 days, while aflibercept was significantly more co-localized after 12 weeks of treatment. Our data indicate that bevacizumab is shuttled outside the cell via exosomes after 1 day of exposure, corresponding to our previous data (Aboul Naga et al. [8]), but after repetitive long-term exposure, more aflibercept is shuttled out of the cells via exosomes. 


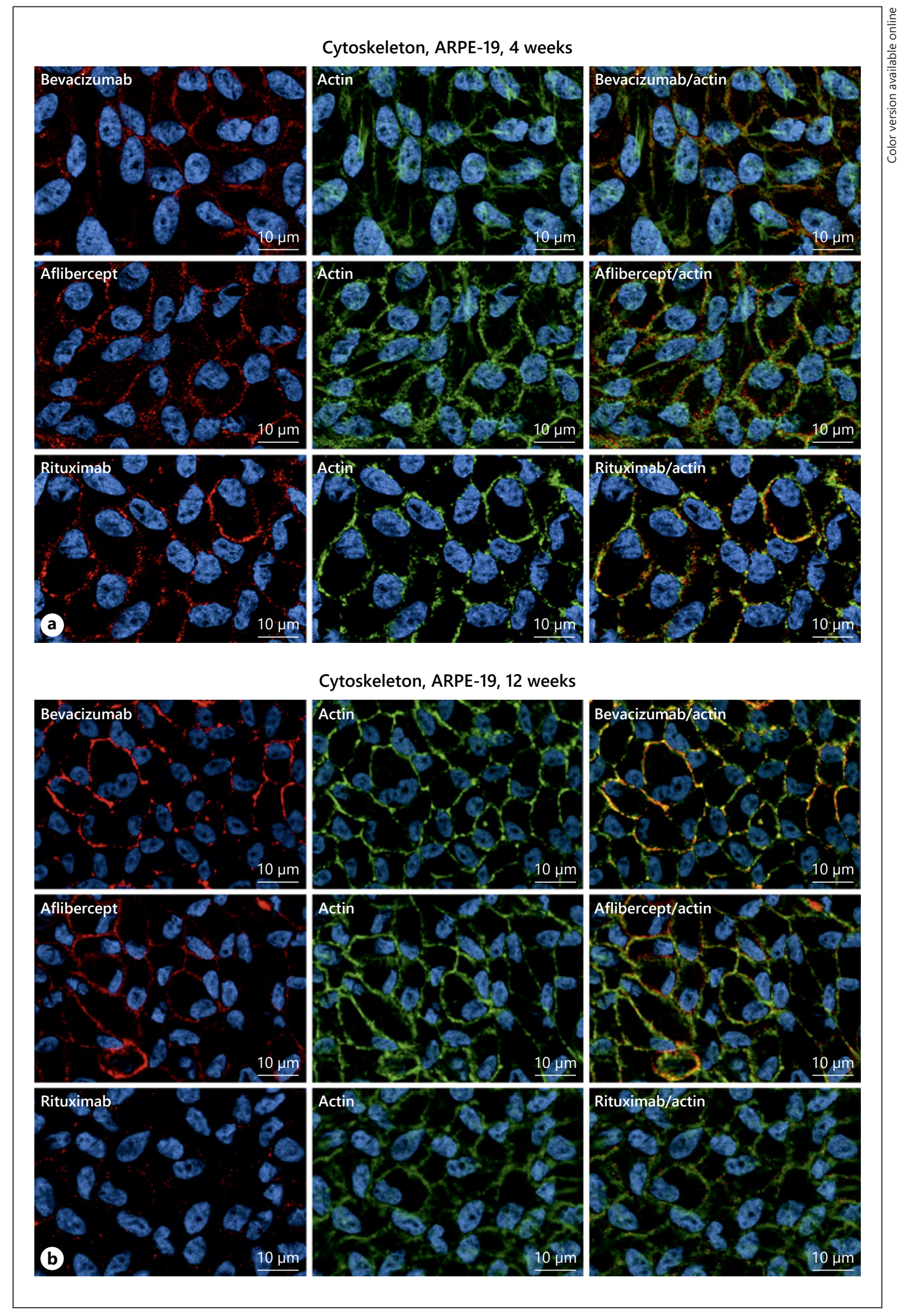

(For legend see next page.) 
In ARPE-19 cells (Fig. 12), significantly more aflibercept was co-localized with CD63 after 7 days and 4 weeks. Contrary to what is found for primary RPE cells, after 12 weeks, significantly more bevacizumab can be found localized with CD63 than aflibercept. In comparison with rituximab, more bevacizumab could be found localized to CD63 at 1 day and 12 weeks of exposure, while after 4 weeks of exposure, more rituximab was co-localized. Aflibercept and rituximab did not display any significant differences in ARPE-19 cells. Of note, the overall co-localization seen with CD63 (for all compounds) was significantly less in ARPE-19 cells compared to primary RPE $(p<0.001)$, a difference that was not seen for Lamp2. Our data indicate differences in the time course of exosomedependent excretion between bevacizumab (early) and aflibercept (long-term) but also between primary RPE and ARPE-19 cells.

\section{Discussion/Conclusion}

The long-term treatment of exudative AMD has become clinical routine. However, the long-term effects of repetitive anti-VEGF application on the retina are not well understood and, especially considering the loss of initial vision gain in many patients after long-term treatment [2], warrant further investigation. VEGF-inhibitors interact with cells of the retina at a cellular level $[6,7,9]$. Therefore, long-term in vitro studies contribute to our understanding of the consequences of long-term antiVEGF treatment on a cellular level.

We have recently introduced a model for repetitive long-term treatment in RPE cell culture, with up to 12 applications of anti-VEGF over a time course of 12 weeks. Moreover, in this in vitro setting, we also model the variation of VEGF concentration by removing the VEGF antagonist after 4 days, allowing the cells to be cultured in a VEGF-containing medium for 3 days [14]. While this is a weekly, not monthly, application, our model does mimic an extended treatment of 12 applications in cell culture, enabling the investigation of repetitious long-term treatment.

Fig. 6. Compounds and cytoskeleton in ARPE-19 cells. Depicted are exemplary pictures of bevacizumab, aflibercept, and rituximab (red) and the actin cytoskeleton (green) after 4 weeks (a) and 12 weeks (b) of treatment. Co-localizations are seen as a yellow/orange signal. At all time points, the compounds show alignments with the cytoskeleton. Cell nuclei are depicted in blue. RPE, retinal pigment epithelium.

Anti-VEGF Long-Term Uptake
In the current study, we used primary RPE cells harvested from porcine eyes [15], as well as ARPE-19 cells [17]. The porcine eye is an excellent model for the human situation, with a diurnal lifestyle reflected in the anatomy of the eye and the retina [20]. Porcine RPE cells, especially when used in first passage, are excellent models of human adult RPE cells [21]. ARPE-19 cells, on the other hand, are of human origin and can also be considered a good model of human RPE cells; however, the limitations have to be considered when interpreting results obtained with these cells [21].

To be able to compare the different compounds, an identical concentration for treatment $(250 \mu \mathrm{g} / \mathrm{mL})$ was chosen. While the presumed clinical concentration of the respective compounds differs $(250-500 \mu \mathrm{g} / \mathrm{mL})[12,22$, 23], $250 \mu \mathrm{g} / \mathrm{mL}$ was chosen as the minimal therapeutic concentration at which any of the compounds is used. We have previously shown that at this concentration VEGF is completely inhibited both by bevacizumab or aflibercept [24].

In our study, we found no toxicity of anti-VEGF compounds even after 12 weeks of treatment, confirming our previous results, where we found no toxicity in this time frame using a lower concentration $(125 \mu \mathrm{g} / \mathrm{mL})$ [14]. Our data suggest safety of these compounds on a cellular level even after repeated and long-term use. One has to keep in mind, however, that the cells treated with anti-VEGF in these studies are healthy cells, while in AMD pathology, RPE cells are challenged by oxidative stress and/or danger signals, inducing a pro-inflammatory milieu. Therefore, further studies need to address the long-term toxicity of these therapeutics when combined with other noxious insults to gain further insights into possible effects of antiVEGF treatment on the viability and function of the RPE, and concomitantly on the development of geographic atrophy.

We have previously shown that both bevacizumab and aflibercept are taken up by RPE (and ARPE-19) cells, transported along the cytoskeleton, and stored in actincovered areas in the cells when investigated in time frames of up to 7 days $[7-9,13]$. In the present study, we extended the duration of treatment to 12 weeks, mimicking long-term treatment. Our study shows that at all time points, intracellular anti-VEGF therapeutics can be found aligned with the cytoskeleton, suggesting transport along them, and intracellular encapsulation, irrespective of the duration of the treatment (though some variation over time may exist). These data indicate that the pathways of intracellular transport and storage do not strongly vary in correlation to treatment duration and are most likely gen- 

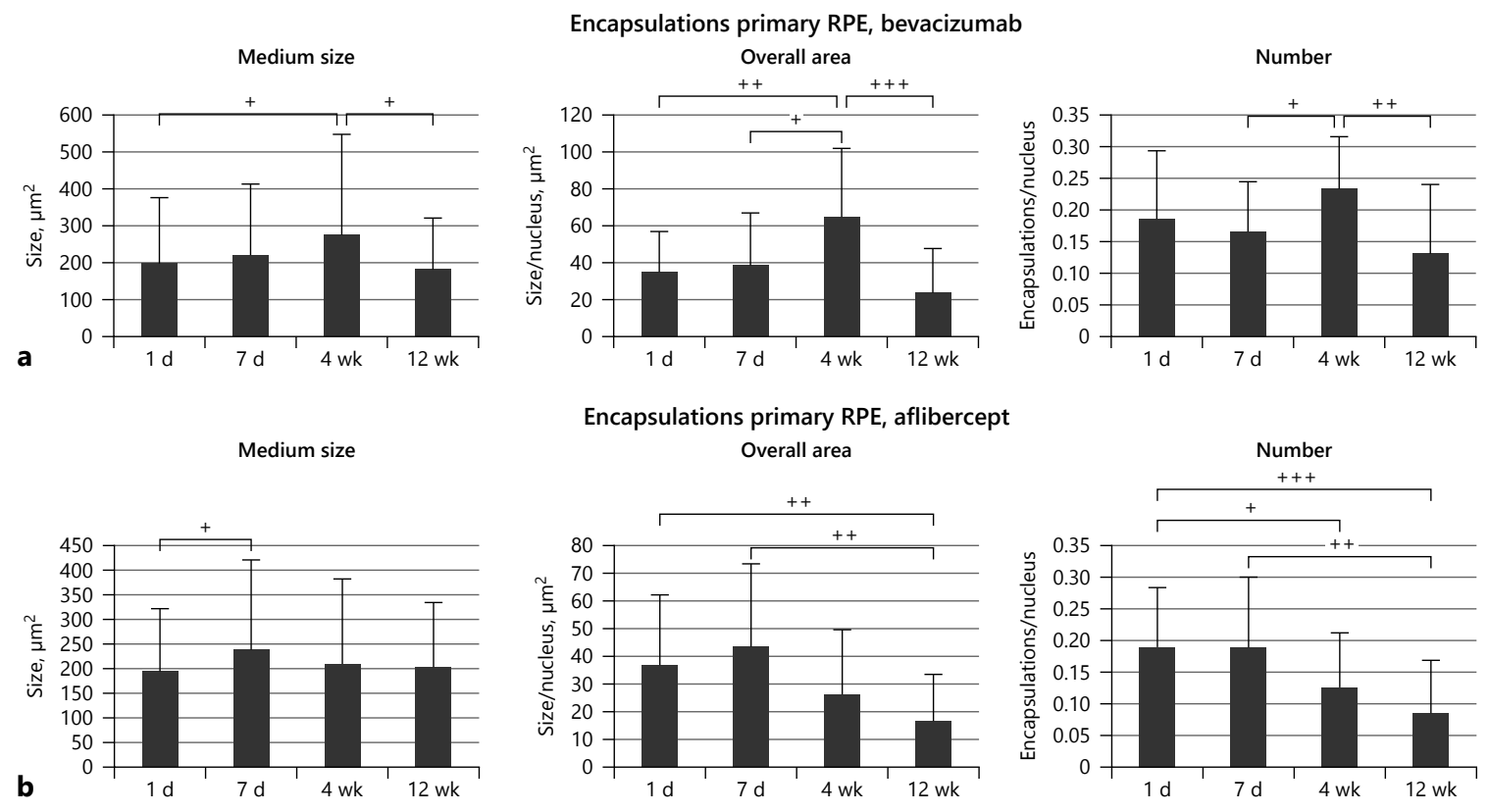

Encapsulations primary RPE, aflibercept
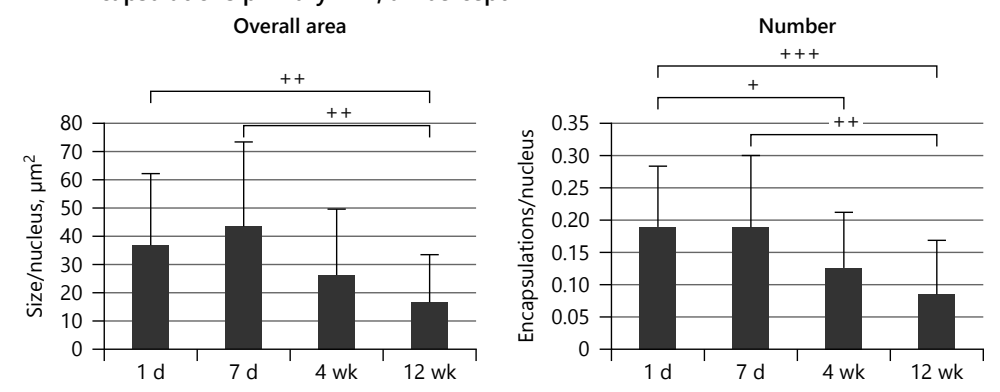

\section{Encapsulations primary RPE, rituximab}

Medium size
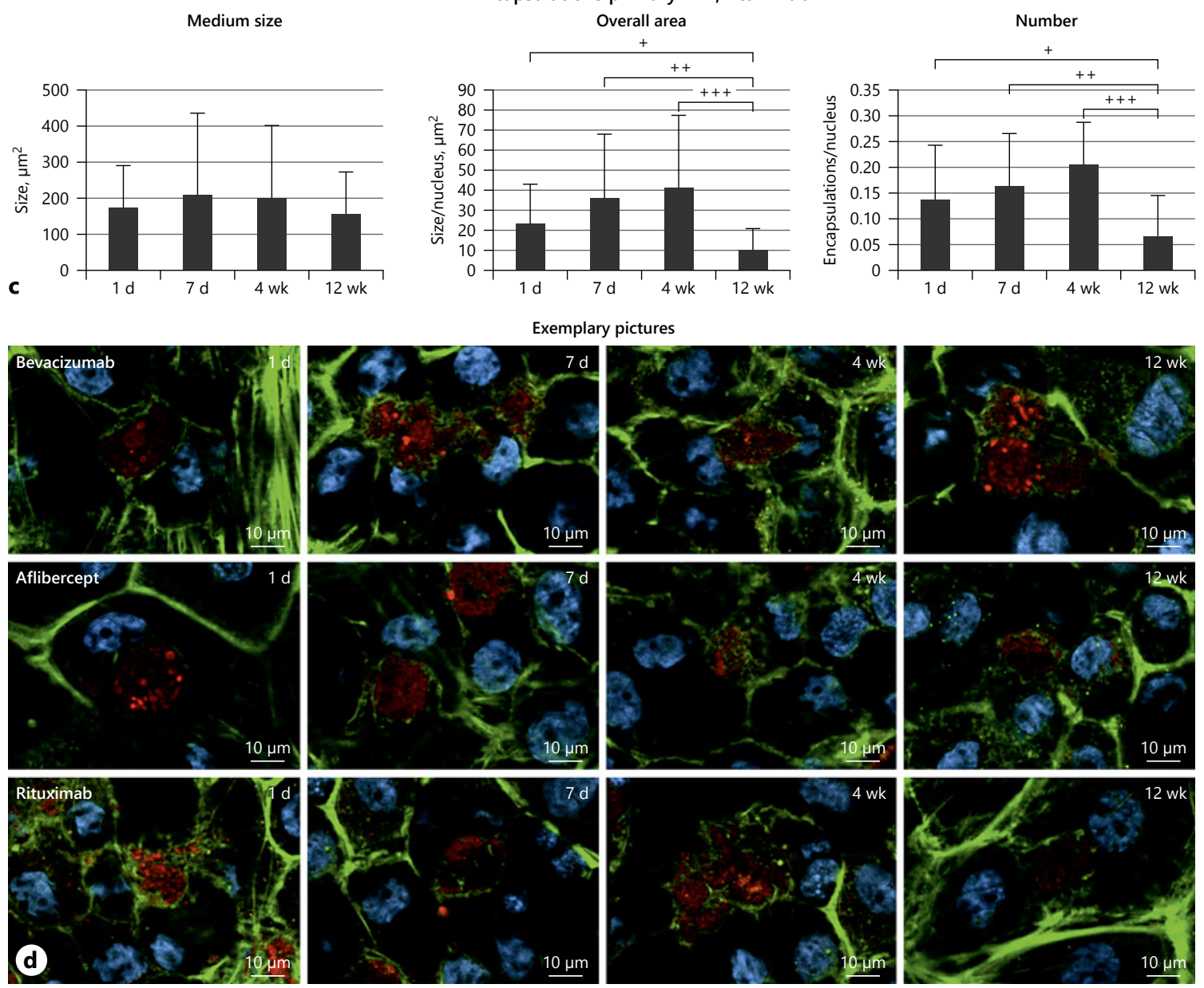

7

(For legend see next page.) 
eral features employed by the RPE for intracellular trafficking of anti-VEGF compounds. Indeed, as rituximab shows the same pattern, it might be a general trafficking pathway for antibodies or, more precisely, Fc-containing molecules.

While these patterns seem to be irrespective of time or compound used, some interesting differences can be found when investigating the strength of the signal of the compound over time and in direct comparison. Of note, the signal has been normalized so that a quantitative analysis of the immunofluorescence was possible. Here, we can see a time-dependent signal which is basically similar for all compounds with the strongest signal found after 1 day, a reduction of the signal seen at 7 days and 4 weeks, and an increase again after 12 weeks. These data suggest that the compound is removed from the cell over time, either by shuttling it out of the cells or by degradation. Our previous data suggested that the compound is transported through the cells and shuttled out of it rather than being degraded, which may be dependent on the Fc-receptor [8, 9]. The finding that compounds can always be found aligned with the actin cytoskeleton can be also interpreted in this direction, as a snapshot of transportation. Of high interest, however, is the direct comparison of the signal of bevacizumab and aflibercept (or rituximab), showing that the bevacizumab signal is much stronger than aflibercept, which can be seen at all time points in the primary RPE (and in most time points in ARPE-19), suggesting a higher uptake (or slower degradation) of bevacizumab. We have shown before that the neonatal Fc-receptor ( FcRn) is involved in the transport of antibodies, but not of aflibercept in RPE cells. Our data further indicated that FcRn is mainly involved in the recycling of antibodies back to the RPE cell [9]. Therefore, the overall reduced signal of aflibercept compared to bevacizumab could be explained by an increased recycling of bevacizumab, but not of aflibercept. For rituximab, which is also transported via FcRn [25], the situation is less clear, as in ARPE-19, we see a stronger signal compared to aflibercept, but in primary RPE cells, the signal is reduced. The situation is, therefore, likely to be more complex than just FcRn recy-

Fig. 7. Encapsulation of bevacizumab (a), aflibercept (b), and rituximab (c) in primary RPE, showing medium size, overall area, and number; Exemplary pictures of encapsulations with compound (red), actin filament (green), and nucleus (blue) (d). Encapsulations were found at all time points for all substances. ${ }^{+} p<0.05$, ${ }^{++} p<0.01,{ }^{+++} p<0.001$. Significance evaluated with Student's $t$ test. d, day; wk, week; RPE, retinal pigment epithelium.

Anti-VEGF Long-Term Uptake cling and influenced by the differences between primary RPE and ARPE-19 cells [21]. When comparing RPE cells and ARPE-19 cells one has to keep in mind that these cells differ in several aspects. While generally considered a valuable RPE model, ARPE-19 displays a less differentiated phenotype, lacks pigmentation, displays more stress fibers (and therefore differ in their distribution of actin filaments), and can differ in their receptor expression, which all may contribute to the differences found in the uptake of the tested biologicals $[8,9,21,26,27]$. Contributing to this complexity is the substance and time-dependent co-localization to exosomes (CD63) in RPE cells, which indicates an early secretion via exosomes for bevacizumab, corresponding to our previous findings [8], but a strong secretion of aflibercept at later time points, with very little association of rituximab with CD63 at all. Again, the results are cell dependent, as in ARPE-19 cells, far fewer co-localizations to CD63 can be found for all compounds and a reversed picture is painted, with more bevacizumab than aflibercept associated with CD63 after 12 weeks. Taken together, our data indicate that exosome secretion may play a part in intracellular reduction of the compounds, but further research is warranted to elucidate the exact role of this mechanism. Another possible explanation for the reduced intracellular signal of aflibercept could be related to degradation of the compound. Indeed, we found significantly more co-localization of aflibercept with Lamp2 compared to bevacizumab in primary RPE cells. This might indicate that RPE cells indeed degrade (some of the) intracellular aflibercept. We have previously shown that aflibercept, but not bevacizumab, is susceptible to degradation by plasmin and that this degradation reduces its ability to inhibit VEGF $[28,29]$. Possibly, the recombinant structure makes aflibercept more vulnerable to degradation in general. As our previous studies also indicate that FcRn might not be involved in the transport of aflibercept through the RPE [9], and FcRn is protecting antibodies from lysosomal degradation [30], it is tempting to speculate that a reduced interaction with $\mathrm{FcRn}$ may be the cause of the reduced intracellular signal of aflibercept compared to bevacizumab. However, this needs further clarification. The question remains whether these differences between bevacizumab and aflibercept may have consequences in the patient. In exudative AMD, choroidal neovascularizations which need to be treated by VEGF antagonists can be found in the retina, in the subretinal space, or beneath the RPE [31]. In the case of the lesion being beneath the RPE (and presuming that the RPE barrier is intact), the VEGF antagonists would have to be transported through the barrier. Here, a reduced uptake 


\section{Encapsulations ARPE-19, bevacizumab}
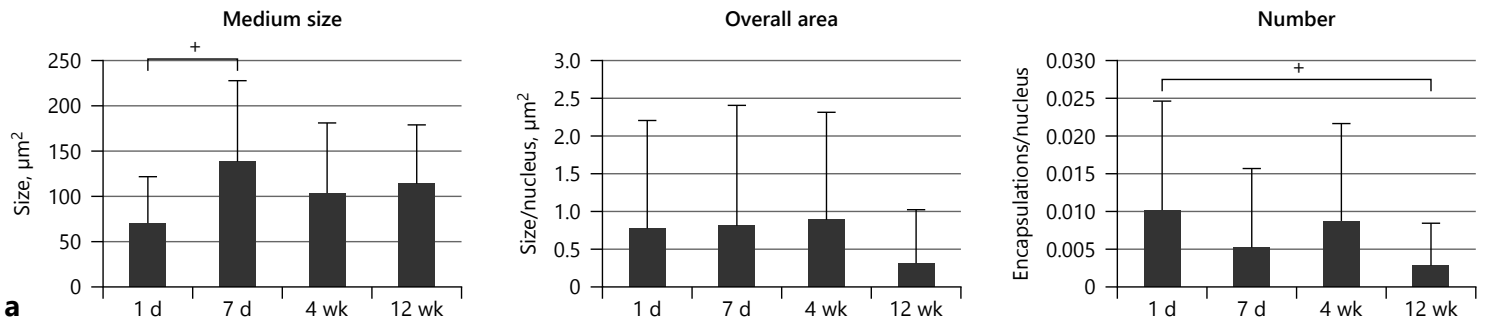

Encapsulations ARPE-19, aflibercept

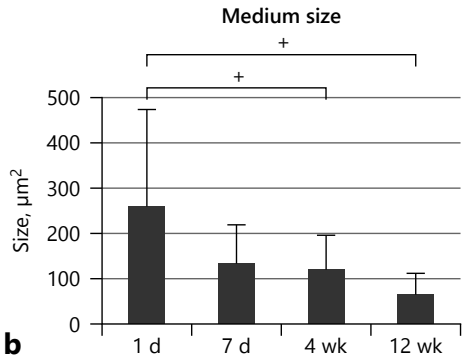

Overall area

Number
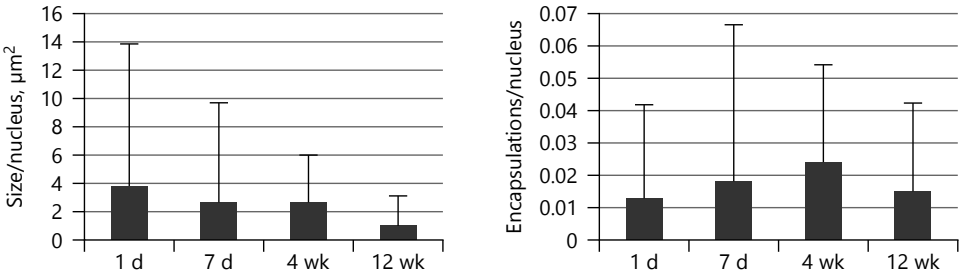

Encapsulations ARPE-19, rituximab
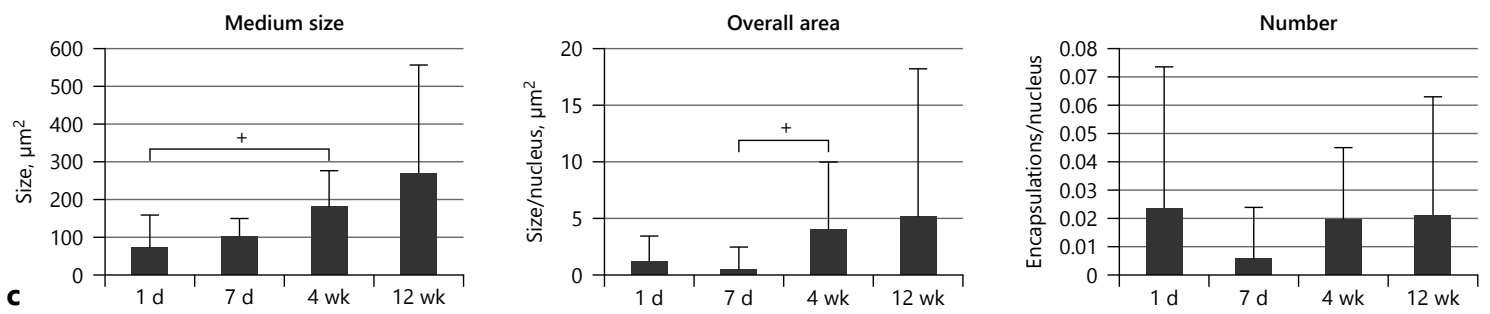

Exemplary pictures
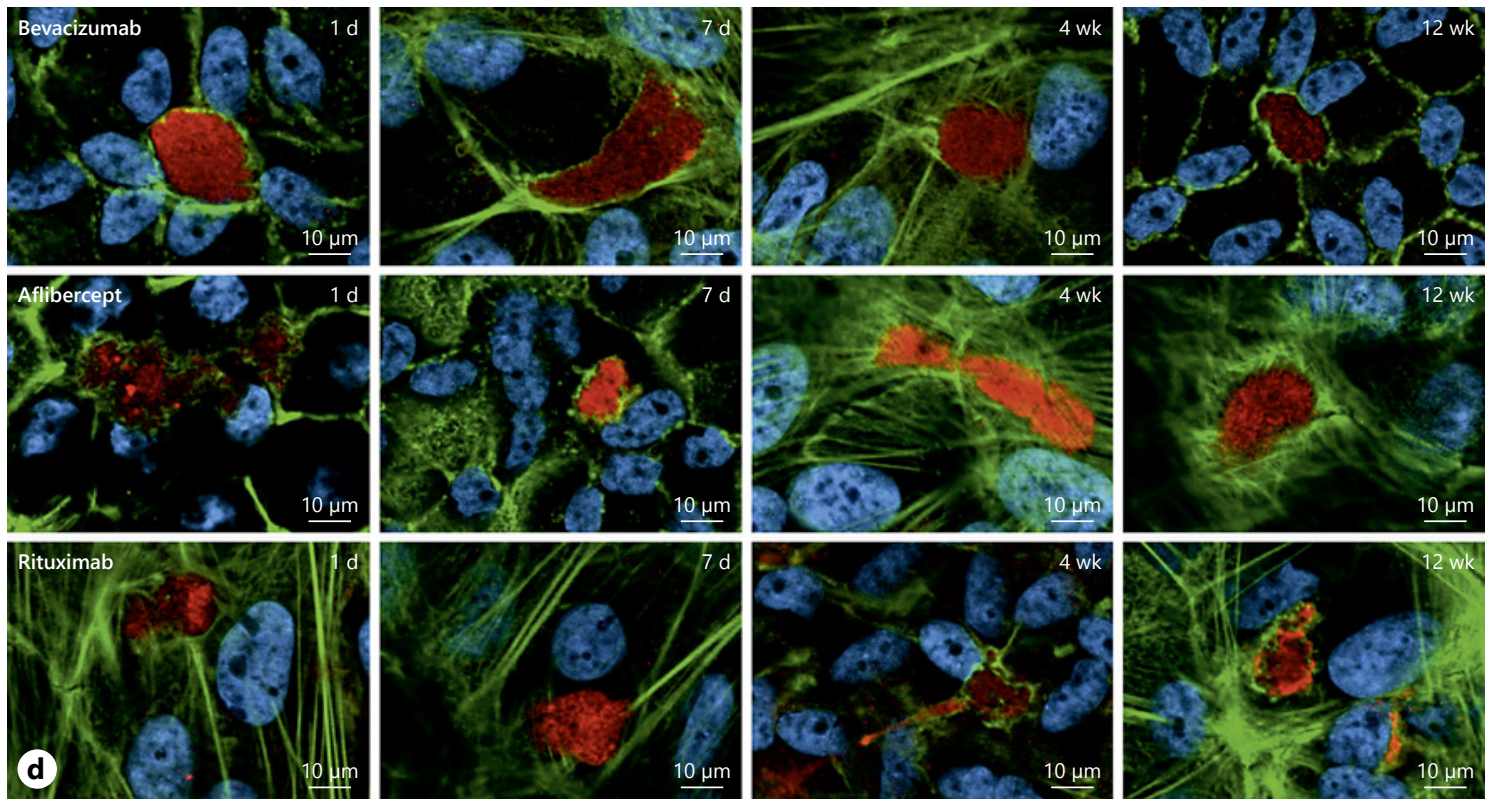

8

(For legend see next page.) 


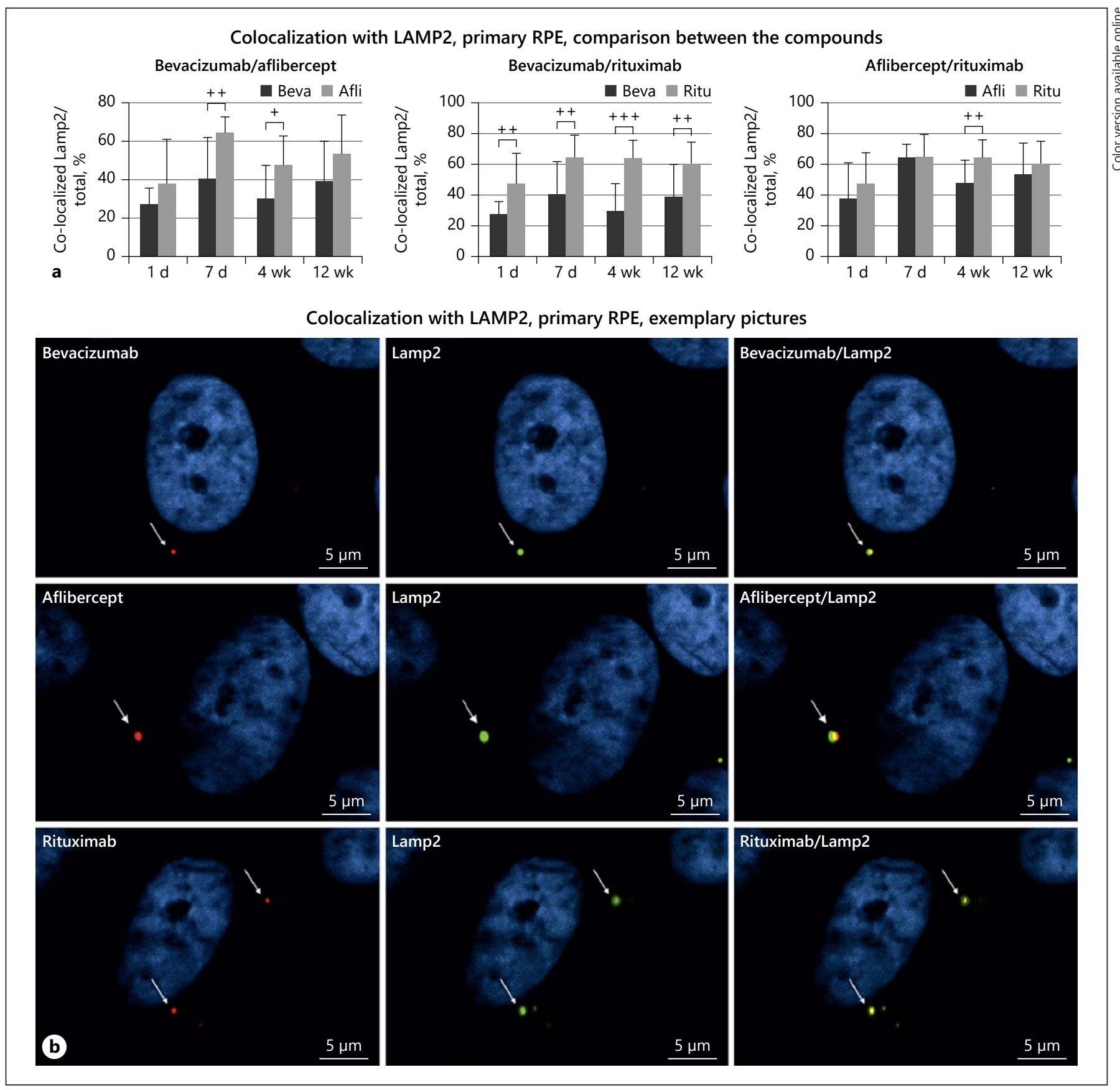

Fig. 9. Relative number of co-localization between Lamp2 and bevacizumab, aflibercept, and rituximab, respectively, in primary RPE. a Comparison of relative number of co-localizations of the different compounds. Bevacizumab displayed less co-localizations with Lamp2 than aflibercept or rituximab. b Exemplary pictures for colocalization in primary RPE, compound (red), Lamp2 (green), co-localization (yellow/orange), and nucleus (blue). ${ }^{+} p<0.05 ;{ }^{++} p<0.01 ;{ }^{+++} p<0.001$. Significance evaluated with Student's $t$ test. Afli, aflibercept; Beva, bevacizmab; Ritu, rituximab; d, day; wk, week; RPE, retinal pigment epithelium.

Fig. 8. Encapsulation of bevacizumab (a), aflibercept (b), and rituximab (c) in ARPE-19, showing medium size, overall area, and number; Exemplary pictures of encapsulations with compound (red), actin filament (green), and nucleus (blue) (d). Encapsulations were found at all time points for all substances. ${ }^{+} p<0.05$. Significance evaluated with Student's $t$ test. d, day; w, week; RPE, retinal pigment epithelium. 


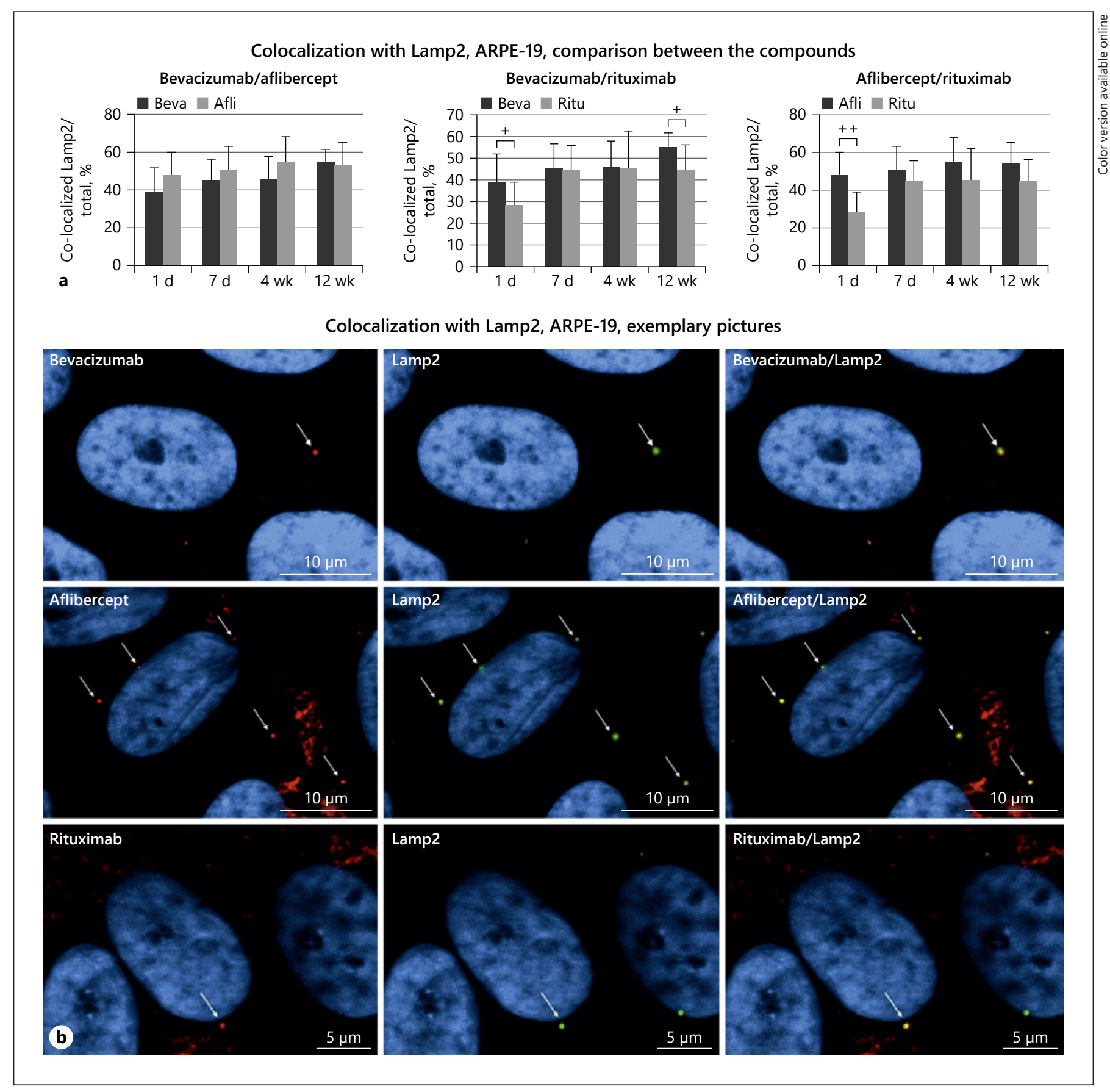

Fig. 10. Relative number of co-localization between Lamp2 and bevacizumab, aflibercept, and rituximab, respectively, in ARPE-19 cells. a Comparison of relative number of co-localizations of the different compounds. No significant differences between bevacizumab and aflibercept can be found. $\mathbf{b}$ Exemplary pictures for co-localization in primary RPE, compound (red), Lamp2 (green), co-localization (yellow/orange), and nucleus (blue). ${ }^{+} p<$ $0.05 ;{ }^{++} p<0.01$. Significance evaluated with Student's $t$ test. Afli, aflibercept; Beva, bevacizmab; Ritu, rituximab; d, day; wk, week; RPE, retinal pigment epithelium. 




Fig. 11. Relative number of co-localization between CD63 and bevacizumab, aflibercept, and rituximab, respectively, in primary RPE. a Comparison of relative number of co-localizations of the different compounds. $\mathbf{b}$ Exemplary pictures for co-localization in primary RPE, compound (red), Lamp2 (green), co-localization (yellow/ orange), nucleus (blue). ${ }^{+} p<0.05 ;{ }^{++} p<0.01 ;{ }^{+++} p<0.001$. Significance evaluated with Student's $t$ test. Afli, aflibercept; Beva, bevacizmab; Ritu, rituximab; d, day; wk, week; RPE, retinal pigment epithelium. 
Colocalization with CD63, ARPE-19, comparison between the compounds
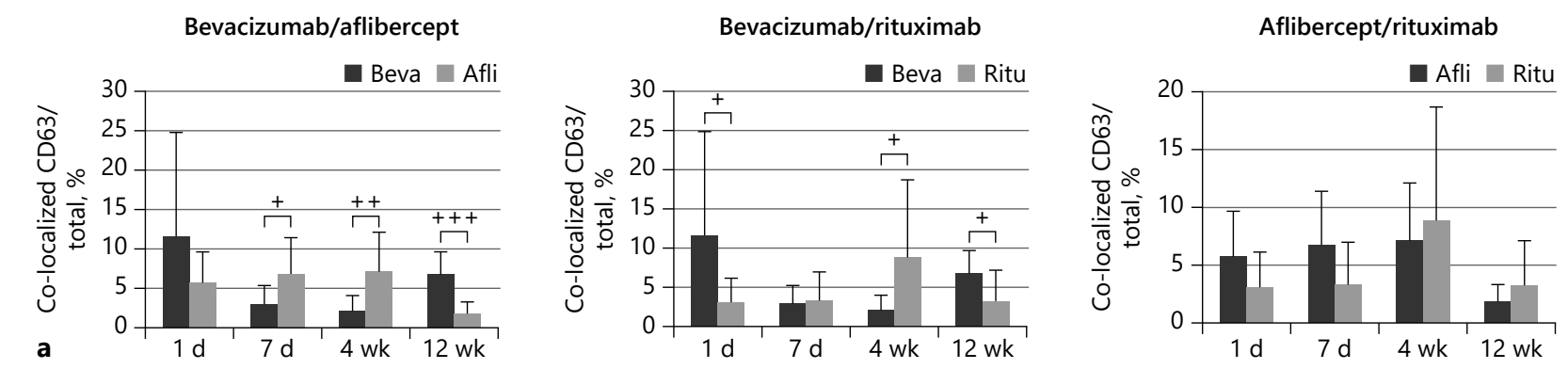

Colocalization with CD63, ARPE-19, exemplary pictures
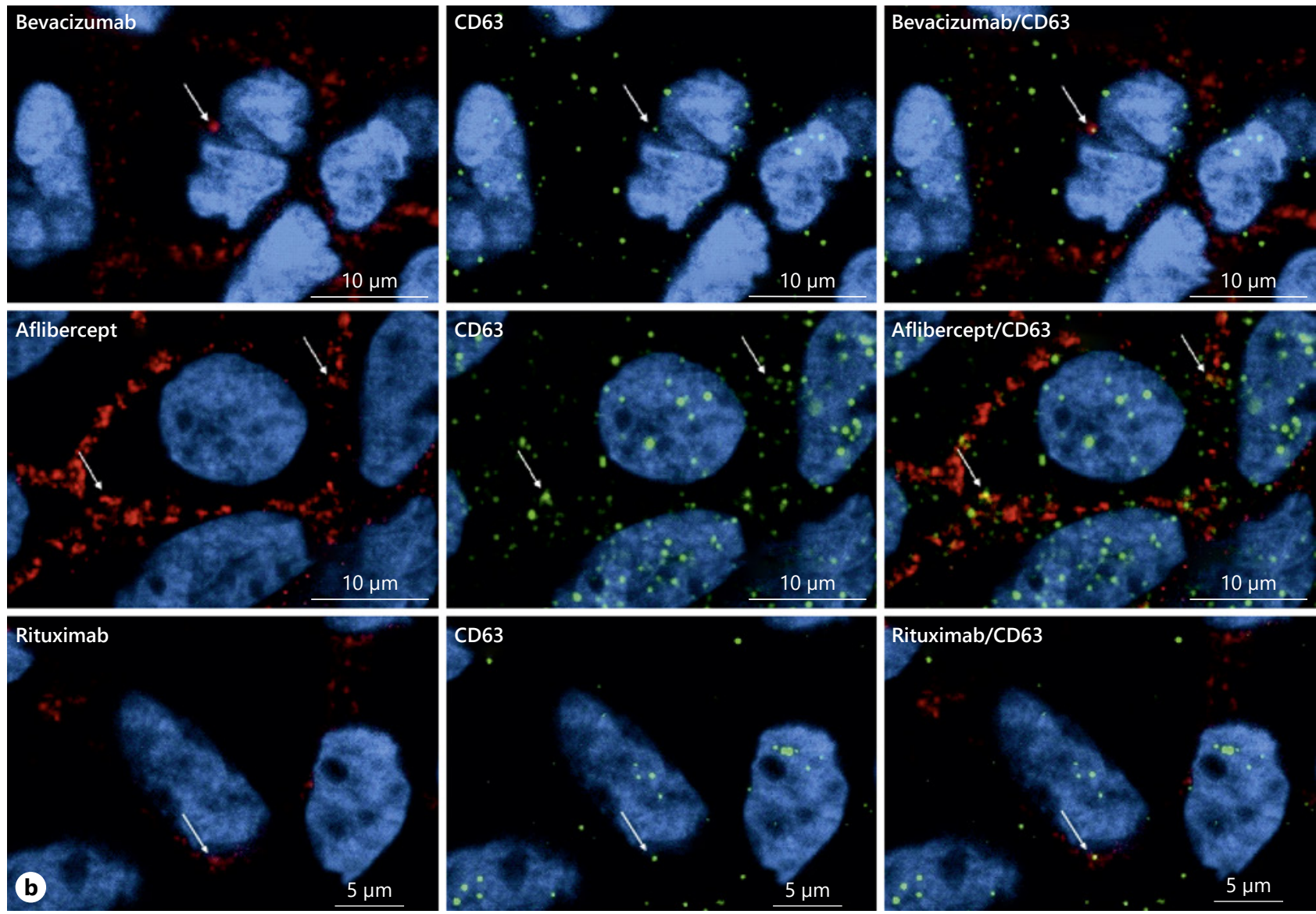

Fig. 12. Relative number of co-localization between CD63 and bevacizumab, aflibercept, and rituximab, respectively, in ARPE-19 cells. a Comparison of relative number of co-localizations of the different compounds. Note that the overall signal is less compared to primary RPE cells. b Exemplary pictures for co-localization in ARPE19, compound (red), Lamp2 (green), co-localization (yellow/orange), nucleus (blue). ${ }^{+} p<0.05 ;{ }^{++} p<0.01$; ${ }^{+++} p<0.001$. Significance evaluated with Student's $t$ test. Afli, aflibercept; Beva, bevacizmab; Ritu, rituximab; d, day; wk, week; RPE, retinal pigment epithelium. 
or enhanced degradation may interfere with the bioavailability of aflibercept at the lesion. Further research is warranted to address this question in more detail and to investigate the consequences of the interaction of the biologicals with retinal cells on their pharmacokinetics and bioavailability in the retina.

Our data show a time and compound-dependent intracellular presence of the biologicals (bevacizumab, aflibercept, and rituximab) in RPE cells in the long-term treatment. We found differences between bevacizumab and aflibercept, indicating a different intracellular processing of the compound and hinting toward a higher availability of bevacizumab. Further research is warranted to further elucidate the interactions of the anti-VEGF compounds with retinal cells and its consequence on bioavailability.

\section{Acknowledgements}

This study was supported by the Stifterverband (HermannWacker foundation). Parts of the study have been presented at the Symposium of the German Ophthalmological Society (DOG), Berlin, 2017.

\section{Statement of Ethics}

This study is exempt from Ethics Committee approval. The material used for this study (porcine eyes) was obtained from the local abattoir, where the animals are killed for the purpose of food production and the eyes are regularly removed from the slaughtered animals due to legal regulations (Tier-LMHV (Anlage $5 \mathrm{zu}$ 17 Satz 2, Kapitel III, No. 2.4). The usage of the eyes for experimental purposes was conducted in agreement with the animal welfare officer of the University of Kiel (Prof. Dr. Gerhard Schultheiß). According to the German animal welfare act (TierSchG), this is not considered to be animal research, but an alternative to the use of animals in research.

\section{Conflict of Interest Statement}

The authors have no conflicts of interest to declare.

\section{Funding Sources}

This study was supported by the Stifterverband (HermannWacker foundation).

\section{Author Contributions}

Conceptualization was done by Alexa Klettner and Johann Roider. Material preparation and data collection were performed by Laura Borchers. Data Analysis was conducted by Laura Borchers and Alexa Klettner. Resources were provided by Johann Roider and Alexa Klettner. The first draft was prepared by Alexa Klettner. All authors read and approved the final manuscript.

\section{References}

1 Schmidt-Erfurth U, Chong V, Loewenstein A, Larsen M, Souied E, Schlingemann R, et al. Guidelines for the management of neovascular age-related macular degeneration by the European Society of Retina Specialists (EURETINA). Br J Ophthalmol. 2014 Sep; 98(9):1144-67.

2 Rofagha S, Bhisitkul RB, Boyer DS, Sadda SR, Zhang K. Seven-year outcomes in ranibizumab-treated patients in ANCHOR, MARINA, and HORIZON: a multicenter cohort study (SEVEN-UP). Ophthalmology. 2013 Nov; 120(11):2292-9.

3 Lois N, McBain V, Abdelkader E, Scott NW, Kumari R. Retinal pigment epithelial atrophy in patients with exudative age-related macular degeneration undergoing anti-vascular endothelial growth factor therapy. Retina. 2013 Jan;33(1):13-22.
4 Young M, Chui L, Fallah N, Or C, Merkur AB, Kirker AW, et al. Exacerbation of choroidal and retinal pigment epithelial atrophy after anti-vascular endothelial growth factor treatment in neovascular age-related macular degeneration. Retina. 2014 Jul;34(7):1308-15.

5 Gerding $\mathrm{H}$. Long-term results of intravitreal anti-VEGF injections in wet AMD: a metaanalysis. Klin Monbl Augenheilkd. 2016 Apr; 233(4):471-4.

6 Klettner A, Möhle F, Roider J. Intracellular bevacizumab reduces phagocytotic uptake in RPE cells. Graefes Arch Clin Exp Ophthalmol. 2010 Jun;248(6):819-24.

7 Klettner A, Tahmaz N, Dithmer M, Richert E, Roider J. Effects of aflibercept on primary RPE cells: toxicity, wound healing, uptake and phagocytosis. Br J Ophthalmol. 2014 Oct; 98(10): 1448-52.

8 Aboul Naga SH, Dithmer M, Chitadze G, Kabelitz D, Lucius R, Roider J, et al. Intracellular pathways following uptake of bevacizumab in RPE cells. Exp Eye Res. 2015 Feb;131:29-41.
9 Dithmer M, Hattermann K, Pomarius P, Aboul Naga SH, Meyer T, Mentlein R, et al. The role of Fc-receptors in the uptake and transport of therapeutic antibodies in the retinal pigment epithelium. Exp Eye Res. 2016 Apr;145:187-205.

10 Ranjbar M, Brinkmann MP, Zapf D, Miura Y, Rudolf M, Grisanti S. Fc receptor inhibition reduces susceptibility to oxidative stress in human RPE cells treated with bevacizumab, but not aflibercept. Cell Physiol Biochem. 2016;38(2):737-47.

11 Vo TA, Abedi S, Schneider K, Chwa M, Kenney MC. Effects of bevacizumab, ranibizum$\mathrm{ab}$, and aflibercept on phagocytic properties in human RPE cybrids with AMD versus normal mitochondria. Exp Eye Res. 2018 Dec; 177:112-6.

12 Spitzer MS, Wallenfels-Thilo B, Sierra A, Yoeruek E, Peters S, Henke-Fahle S, et al. Antiproliferative and cytotoxic properties of bevacizumab on different ocular cells. Br J Ophthalmol. 2006 Oct;90(10):1316-21. 
13 Klettner AK, Kruse ML, Meyer T, Wesch D, Kabelitz D, Roider J. Different properties of VEGF-antagonists: bevacizumab but not ranibizumab accumulates in RPE cells. Graefes Arch Clin Exp Ophthalmol. 2009 Dec; 247(12):1601-8.

14 Schottler J, Randoll N, Lucius R, Caliebe A, Roider J, Klettner A. Long-term treatment with anti-VEGF does not induce cell aging in primary retinal pigment epithelium. Exp Eye Res. 2018 Jun;171:1-11.

15 Wiencke AK, Kiilgaard JF, Nicolini J, Bundgaard M, Röpke C, La Cour M. Growth of cultured porcine retinal pigment epithelial cells. Acta Ophthalmol Scand. 2003 Apr;81(2): 170-6.

16 Klettner A, Roider J. Comparison of bevacizumab, ranibizumab, and pegaptanib in vitro: efficiency and possible additional pathways. Invest Ophthalmol Vis Sci. 2008 Oct;49(10): 4523-7.

17 Dunn KC, Aotaki-Keen AE, Putkey FR, Hjelmeland LM. ARPE-19, a human retinal pigment epithelial cell line with differentiated properties. Exp Eye Res. 1996 Feb;62(2):15569.

18 Riss TL, Moravec RA, Niles AL, Duellman S, Benink HA, Worzella TJ, et al. Cell viability assays. In: Sittampalam GS, Grossman A, Brimacombe K, Arkin M, Auld D, Austin C, et al., editors. Assay guidance manual. Bethesda, MD: Eli Lilly \& Company and the National Center for Advancing Translational Sciences; 2004.
19 Hillenkamp J, Dydykina S, Klettner A, Treumer F, Vasold R, Bäumler W, et al. Safety testing of indocyanine green with different surgical light sources and the protective effect of optical filters. Retina. 2010 Nov-Dec; 30(10):1685-91.

20 Middleton S. Porcine ophthalmology. Vet Clin North Am Food Anim Pract. 2010 Nov; 26(3):557-72.

21 Klettner A. Retinal pigment rpithelium cell culture. In: Klettner A, Dithmar S, editors. Retinal pigment epithelium in health and disease. Switzerland: Springer Nature; 2020. p. 295-306.

22 Malik D, Tarek M, Caceres del Carpio J, Ramirez C, Boyer D, Kenney MC, et al. Safety profiles of anti-VEGF drugs: bevacizumab, ranibizumab, aflibercept and ziv-aflibercept on human retinal pigment epithelium cells in culture. Br J Ophthalmol. 2014 Jun;98(Suppl 1):i11-16.

23 Sobolewska B, Heiduschka P, Bartz-Schmidt $\mathrm{KU}$, Ziemssen F. pH of anti-VEGF agents in the human vitreous: low impact of very different formulations. Int J Retina Vitreous. 2017; $3: 22$.

24 Klettner A, Recber M, Roider J. Comparison of the efficacy of aflibercept, ranibizumab, and bevacizumab in an RPE/choroid organ culture. Graefes Arch Clin Exp Ophthalmol. 2014 Oct;252(10):1593-8.

25 Dithmer M, Fuchs S, Shi Y, Schmidt H, Richert E, Roider J, et al. Fucoidan reduces secretion and expression of vascular endothelial growth factor in the retinal pigment epithelium and reduces angiogenesis in vitro. PloS One. 2014;9(2):e89150.
26 Geisen P, McColm JR, King BM, Hartnett ME. Characterization of barrier properties and inducible VEGF expression of several types of retinal pigment epithelium in medium-term culture. Curr Eye Res. 2006 Sep; 31(9):739-48.

27 van Bilsen K, van Hagen PM, Bastiaans J, van Meurs JC, Missotten T, Kuijpers RW, et al. The neonatal Fc receptor is expressed by human retinal pigment epithelial cells and is downregulated by tumour necrosis factor-alpha. Br J Ophthalmol. 2011 Jun;95(6):864-8.

28 Klettner A, Puls S, Treumer F, Roider J, Hillenkamp J. Compatibility of recombinant tissue plasminogen activator and bevacizumab co-applied for neovascular age-related macular degeneration with submacular hemorrhage. Arch Ophthalmol. 2012 Jul;130(7): 875-81.

29 Klettner A, Grotelüschen S, Treumer F, Roider J, Hillenkamp J. Compatibility of recombinant tissue plasminogen activator (rtPA) and aflibercept or ranibizumab coapplied for neovascular age-related macular degeneration with submacular haemorrhage. $\mathrm{Br} \mathrm{J} \mathrm{Oph-}$ thalmol. 2015 Jun;99(6):864-9.

30 Simister NE, Jacobowitz Israel E, Ahouse JC, Story CM. New functions of the MHC class I-related Fc receptor, FcRn. Biochem Soc Trans. 1997 May;25(2):481-6.

31 Cohen SY, Creuzot-Garcher C, Darmon J, Desmettre T, Korobelnik JF, Levrat F, et al. Types of choroidal neovascularisation in newly diagnosed exudative age-related macular degeneration. Br J Ophthalmol. 2007 Sep; 91(9):1173-6. 